\title{
Crisis, ingresos y mercado de trabajo en Ecuador
}

Crise, revenus et marché du travail en Équateur

Crisis, Income and labor market in Ecuador

Dante Contreras y María Luisa Granda

\section{OpenEdition}

Journals

Edición electrónica

URL: http://journals.openedition.org/bifea/6789

DOI: $10.4000 /$ bifea.6789

ISSN: 2076-5827

\section{Editor}

Institut Français d'Études Andines

Edición impresa

Fecha de publicación: 1 diciembre 2002

Paginación: 621-654

ISSN: 0303-7495

Referencia electrónica

Dante Contreras y María Luisa Granda, « Crisis, ingresos y mercado de trabajo en Ecuador », Bulletin de I'Institut français d'études andines [En línea], 31 (3) | 2002, Publicado el 08 diciembre 2002, consultado el 08 diciembre 2020. URL : http://journals.openedition.org/bifea/6789; DOI : https://doi.org/10.4000/ bifea.6789

\section{(c) (i) $\odot$}

Les contenus du Bulletin de l'Institut français d'études andines sont mis à disposition selon les termes de la licence Creative Commons Attribution - Pas d'Utilisation Commerciale - Pas de Modification 4.0 International. 


\title{
CRISIS, INGRESOS Y MERCADO DE TRABAJO EN ECUADOR
}

\author{
Dante CONTRERAS, María Luisa GRANDA*
}

\section{Resumen}

Ecuador ha atravesado una profunda crisis económica y política los últimos años. Los indicadores de desigualdad, cuyos determinantes se analizan con profundidad en este trabajo, muestran variaciones entre 1995 y 1998. La evidencia indica que los factores más significativos para explicar la desigualdad son la escolaridad, la pertenencia al sector agrícola, el género y el grado de formalidad del empleo. Al desagregar la muestra por estas características, se encuentran diferencias en la forma en que cada uno de estos factores contribuyen a la desigualdad. La explicación se encontraría en que se han dado cambios en la estructura productiva de la familia, entre los cuales destacan la forma de participación de la mujer y un proceso migratorio al extranjero que se aceleró justamente a partir de 1998.

Palabras clave: Retornos a la educación, género, discriminación salarial, desigualdad de ingresos.

\section{CRISE, REVENUS ET MARCHÉ DU TRAVAIL EN ÉQUATEUR}

\section{Résumé}

Au court des dernières qannées, l'Équateur a traversé une profonde crise économique et politique. Les indicateurs d'inégalité, dont les déterminants sont sanalysés à fond dans ce travail, montrent des changements entre 1995 et 1998. Les données indiquent que les facteurs les plus significatifs qui expliquent l'inégalité sont : la scolarité, l'appartenance au secteur agricole, le sexe et le degré de formalité de l'emploi. En désagrégant ces caracteristiques de l'e'chantillon, les différentes contributions de chaque facteur à l'inégalité se manifestent. La raison pourrait en être les changements survenus dans la structure productive de la famille, parmi lesqueles se trouvent la participation des femmes et le phénomène migratoire, qui s'est accentué depuis 1998.

Mots clés : Retornos a la educación, género, discriminación salarial, desigualdad de ingresos.

\section{CRISIS, INCOME AND LABOR MARKET IN ECUADOR}

\begin{abstract}
In recent years, Ecuador has gone through a severe economic and political crisis. Inequality indicators for the 1995-1998 period, which are analized in depth in this paper, exhibit

* Por orden alfabético: Departamento de Economía, Universidad de Chile. Instituto de Ciencias Humanísticas y Económicas, Escuela Superior Politécnica del Litoral. E-mail: dcontrer@econ.uchile.cl; mgranda@goliat.espol.edu.ec.
\end{abstract}


a rising trend. The evidence indicates that the most significant factors that contribute to inequality are education, belonging to the agricultural sector, gender, and the degree of job formality. Disaggregating the sample according to these characteristics shows that different sources of inequality are found in each group. This may be due to changes in the productive structure of families, such as, the female participation and migration process, which increased in 1998.

Palabras clave: Return to schooling, gender, wage discrimantion, earnings inequality.

\section{INTRODUCCIÓN}

Ecuador destaca en América Latina por las ingentes condiciones de pobreza y desigualdad de ingresos de sus habitantes (1). Esta situación es mucho más relevante desde 1998 en que se profundizó una crisis económica y política, que habría tenido efectos severos sobre la distribución de ingresos. Este artículo contribuye a analizar los principales factores que explican el nivel de desigualdad y sus cambios en el período previo a la crisis.

Los métodos de descomposición de Shorrocks (1983) y Fields \& Yoo (2000) se utilizan para identificar los determinantes de la desigualdad. De este modo, los índices de desigualdad son desagregados en contribuciones porcentuales, que constituyen una medida del poder explicativo de las distintas fuentes que generan inequidad.

Durante el período analizado 1995-1998, la evidencia muestra un aumento en el nivel de desigualdad, aun cuando las fuentes que la generan mantienen su contribución relativa. Dichas fuentes son principalmente las diferencias en escolaridad, la discriminación por género, el grado de formalidad del empleo del individuo y la participación en el sector agrícola. Esta evidencia sirve de sustento para separar la muestra en función de estas características y examinar con mayor profundidad cada grupo.

La descomposición por fuentes de ingreso familiar sugiere que la crisis tuvo un efecto significativo al interior de los grupos. La explicación se encontraría en que se han dado cambios en la estructura productiva de la familia de manera que las mismas fuentes de desigualdad varían su contribución en cada año. Entre estos cambios destacan la participación de la mujer y un proceso migratorio al extranjero que se aceleró justamente a partir de 1998.

El trabajo está organizado de la siguiente manera. En la próxima sección se realiza un breve análisis del contexto macroeconómico de Ecuador en el período de estudio. La sección 2 presenta la metodología utilizada. La sección 3 muestra los resultados del análisis de descomposición preliminar y en la sección 4 se explora a fondo los determinantes de la desigualdad para cada uno de los grupos antes citados. Finalmente, se delinean las principales conclusiones en la última sección.

(1) Según el BID (1999), Ecuador tiene un coeficiente de Gini de 0,57; sólo Brasil y Paraguay presentan mayor desigualdad, con un coeficiente de Gini de 0,60. En conjunto con la desigualdad, la pobreza creció de 34\% a 71\% entre 1995 y 2000. 


\section{CONTEXTO ECONÓMICO: DESIGUALDAD}

Ecuador tiene una economía basada principalmente en recursos naturales, con estructuras de demanda y oferta relativamente rígidas y gran parte de su fuerza laboral con bajo nivel de escolaridad. La última década ha sido caracterizada como un período de inestabilidad económica y política, culminando en la crisis que alcanzó su momento más difícil en 1999, año en que el PIB real cayó en 7,3 puntos porcentuales. La dolarización de la economía fue la opción para superar la crisis y aun cuando la percepción actual de su desempeño es satisfactoria (2), no se deja de lado la necesidad de implementar una serie de reformas que permitan continuar con éxito la recuperación iniciada. Entre ellas, la reactivación del sistema bancario, la reestructuración del sistema de seguridad social y la conclusión del proceso de privatizaciones.

Desde 1992, se inició un importante ciclo de reformas y liberalización económica que logró controlar la inflación y reducir el tamaño del estado. A partir de 1995, comienza una serie de eventos de orden político, financiero (3) y shocks naturales (4) que inmediatamente se transmiten al ámbito económico. Posteriormente la recuperación se dificulta por la inestabilidad de los gobiernos (5) y la falta de un programa sostenido con este objetivo. Aunque a principios de 1998 la economía había empezado a mejorar, la recuperación fue leve y no sostenida.

El empleo y las condiciones laborales fueron duramente golpeadas por la crisis, llevando a un incremento en el nivel de desigualdad. Al igual que otras economías en desarrollo, en Ecuador se observa elevadas tasas de subempleo, las cuales han persistido la última década. Entre 1995 y 2000, el subempleo aumentó del 45\% al 66\% (6). El fenómeno migratorio se aceleró como resultado de la crisis. Se estima que alrededor de trescientos mil ecuatorianos (8\% de la población económicamente activa) habría abandonado el país en busca de oportunidades de empleo entre 1998 y 2000 (7).

La pobreza también se ha visto acrecentada, producto de la crisis. Según León (2001), la pobreza en 1995 habría alcanzado al 31,8\% de la población, proporción que en 1998 aumentó a 37,9\%. (8)

(2) A partir del 2000, la tasa de crecimiento del producto ha sido positiva. Sin embargo, todavía persisten altas tasas de inflación.

(3) 1995 se inició con la quiebra de uno de los bancos más importantes del país y su posterior rescate por parte del Estado. Ese fue el primero de una serie de bancos que el Estado asumió a su cargo. El sistema financiero todavía no se encuentra en normal funcionamiento.

(4) La economía ecuatoriana se ha visto constantemente afectada por el Fenómeno del Niño. En 1998, esto generó una crisis eléctrica que mantuvo restricciones en la electricidad durante siete meses consecutivos, serios daños en infraestructura y pérdidas en la producción agrícola. Los daños se cuantificaron en alrededor del 17\% del Producto Interno Bruto.

(5) Entre 1995 y 1999, Ecuador tuvo 5 diferentes presidentes de la República.

(6) Encuestas Urbanas de Empleo, Instituto Nacional de Estadísticas y Censos.

(7) Existen diversas fuentes para el número de migrantes al extranjero, pero ninguna cifra oficial. Ver Acosta (2001); Eguez (2001); León (2001).

(8) A diferencia de estos resulados, en este artículo se estimó que el 27\% de los individuos se encontrarían bajo la línea de pobreza en ambos períodos. 
La pregunta de cuál fue el efecto de la crisis sobre la desigualdad se ha intentado responder con distintos argumentos. Jácome et al. (1998) sugieren que el aumento de la desigualdad (9) en el período 1988-1998, fue causado por la caída del ingreso real y del crecimiento del sector informal de la economía. Estos resultados tienen la limitación de ser realizados a base de información para el sector urbano y con datos poco confiables (10). De Jong \& Vos (2000) consideran el efecto del empleo, la participación y la estructura salarial sobre la desigualdad. Postulan que estas variables habrían provocado cambios en la inequidad en el período 1990-1995 (considerado un período de estabilización) y la segunda mitad de la década (período de depresión macroeconómica). Algunas de sus conclusiones difieren de los resultados del presente trabajo, como se mostrará posteriormente.

Este artículo responde la pregunta ¿qué factores microeconómicos específicos contribuyen a explicar los niveles y los cambios en desigualdad en el período analizado? La contribución del mismo es analizar elementos que antes no se consideran en el estudio de la desigualdad como la escolaridad, la pertenencia al sector agrícola y la discriminación por género. El nivel de educación explica alrededor del $40 \%$ del nivel de desigualdad, mientras que el ser trabajador agrícola es el factor con mayor variación, pasando de explicar cerca del $20 \%$ en 1995 al 12\% en 1998. Las condiciones prevalecientes en el mercado de trabajo debido a la crisis, como cambios en la decisión y forma de participación femenina y la migración al extranjero así como los shocks naturales serían los canales de transmisión de la crisis en desigualdad.

\section{METODOLOGÍA}

Este artículo examina la desigualdad y sus cambios a través de la metodología de descomposición propuesta por Fields y Shorrocks, métodos que permiten desagregar los indicadores de desigualdad en función de sus principales determinantes.

\section{1. Descomposición de Fields}

Fields \& Yoo (2000) utilizan la ecuación de Mincer como base para descomponer un índice de desigualdad de ingresos. La medida de desigualdad utilizada es la varianza del logaritmo del ingreso individual. Es decir, se estima la siguiente ecuación:

$$
\ln Y=\sum_{i=1}^{I} \beta_{i} x_{i}=\beta_{0}+\beta_{1} x_{1}+\beta_{2} x_{2}+\ldots+\beta_{I} x_{I}+u_{i}
$$

donde $\ln \mathrm{Y}$ es el logaritmo del ingreso, $\beta_{\mathrm{i}}$ son los coeficientes asociados a cada factor $\mathrm{i}, \mathrm{y} ; \mathrm{x}_{\mathrm{i}}$ son los factores que explican el ingreso.

(9) Muestra un incremento del Gini urbano de 0,46 en 1990 a 0,50 en 1998.

(10) Los propios autores destacan que la medición de la desigualdad se puede tomar sólo como un indicador, dadas las limitaciones de las variables utilizadas para medirlas, como por ejemplo ingresos primarios. Según ellos se subestima el ingreso del hogar en un grado significativo. Para mayores referencias respecto a este punto, ver el apéndice de Jácome et al. (1998). 
Según esta descomposición, la varianza del logaritmo del ingreso (medida de desigualdad) está explicada en principio por los mismos factores que explican el (nivel de) ingreso y cada uno de ellos contribuye en diferentes magnitudes y sentidos a la desigualdad.

Para derivar la fórmula de las contribuciones se parte de la siguiente definición. La covarianza del logaritmo del ingreso consigo mismo puede expresarse como

$$
\operatorname{cov}\left[\sum_{i=1}^{I} \beta_{i} x_{i} ; \ln Y\right]=\sum_{i=1}^{I} \operatorname{cov}\left[\beta_{i} x_{i} ; \ln Y\right]
$$

dado que el término del lado izquierdo corresponde a la varianza del logaritmo del ingreso, por ser la covarianza del logaritmo del ingreso consigo mismo, se genera la siguiente descomposición de varianza.

$$
\sigma^{2}(\ln Y)=\sum_{i=1}^{I} \operatorname{cov}\left[\beta_{i} x_{i} ; \ln Y\right]
$$

al dividir ambos lados de la ecuación para la varianza del logaritmo del ingreso, se tiene

$$
100 \%=\frac{\sum_{i=1}^{I} \operatorname{cov}\left[\beta_{i} x_{i} ; \ln Y\right]}{\sigma^{2}(\ln Y)} \equiv \sum_{i=1}^{I} s_{i}
$$

La descomposición de Fields consiste en computar cada valor $\mathrm{s}_{\mathrm{i}}$, contribución del factor i en términos porcentuales como

$$
s_{i}=\frac{\operatorname{cov}\left[\beta_{i} x_{i} ; \ln Y\right]}{\sigma^{2}(\ln Y)}=\frac{\beta_{i} \cdot \sigma\left(x_{i}\right) \cdot \operatorname{corr}\left[x_{i} ; \ln Y\right]}{\sigma(\ln Y)}
$$

La contribución de un factor depende del coeficiente de la ecuación de ingresos asociado al factor i, de la desviación estándar del factor i y de la correlación entre el factor i y el logaritmo del ingreso. La suma de las contribuciones de cada factor i al ingreso suman $100 \%$, incluyendo la parte no explicada que corresponde al residuo de la ecuación de Mincer.

$$
100 \%=\sum_{i} s_{i}[\ln Y]
$$

Con esta metodología se computan las contribuciones para Ecuador considerando en el análisis factores como la educación, la experiencia, el género y otras características familiares y del trabajo del individuo. Los resultados son presentados en la siguiente sección. 


\section{2. Descomposición de Shorrocks}

El ingreso total de un hogar puede ser desagregado en los montos recibidos de una variedad de fuentes (11). Es factible evaluar el impacto de cada una en la distribución conjunta de ingresos, computando contribuciones a la desigualdad.

Sea $\mathrm{Y}=\mathrm{Y}_{1}, \mathrm{Y}_{2}, \ldots, \mathrm{Y}_{\mathrm{n}}$ la representación para la distribución de ingreso total $\mathrm{Y}$, donde el subíndice representa al individuo i. Y sea $\mathrm{Y}_{\mathrm{k}}=\mathrm{Y}_{1 \mathrm{k}}, \mathrm{Y}_{2 \mathrm{k}}, . ., \mathrm{Y}_{\mathrm{nk}}$ la representación para la distribución de ingreso de cada fuente $\mathrm{k}$.

Si se define I(.) como una medida de la desigualdad de ingresos (12),

$$
I(Y) \leq \sum_{k} \frac{\mu_{k}}{\mu} I\left(Y_{k}\right)
$$

es una función convexa y no negativa de los ingresos relativos, donde $\mu_{\mathrm{k}}$ es la media del ingreso proveniente del factor $\mathrm{k} \mathrm{y} \mu$ es la media del ingreso total.

El objetivo de la metodología es descomponer la medida de desigualdad, de manera que la sumatoria en $\mathrm{k}$ de las contribuciones $\left(\mathrm{S}_{\mathrm{k}}\right)$ de cada una de las $\mathrm{k}$ fuentes, sea igual al valor del índice de desigualdad para el ingreso agregado. Esto es:

$$
I(Y)=\sum_{k} S_{k}
$$

Estas contribuciones son no negativas, aunque se puede dar el caso de un factor igualizante más que contribuyente a la desigualdad. Adicionalmente, se puede calcular las contribuciones proporcionales o relativas, (que suman uno) y son útiles para efectos de comparación. Por ejemplo, si hubiera dos fuentes principales, como ingresos laborales y no laborales, la contribución que hace cada una al índice se puede representar en nivel, donde deberían sumar el valor del índice, o en términos porcentuales, donde deberían sumar uno. Se define la contribución proporcional del factor $\mathrm{k}\left(\mathrm{s}_{\mathrm{k}}\right)$ como

$$
s_{k}=\frac{S_{k}}{I(Y)}
$$

Cualquier función que genere valores adecuados de $\mathrm{s}_{\mathrm{k}}$ (tal que $\sum_{\mathrm{k}} \mathrm{s}_{\mathrm{k}}=1$ ) será una regla de descomposición. La limitación principal de esta metodología, es que la regla de descomposición no es única; dependerá de la fórmula utilizada para el índice de desigualdad. Shorrocks (1982) muestra la existencia de una única regla de descomposición.

(11) Se asume que las fuentes que componen el ingreso son mutuamente excluyentes, y que el ingreso total es la suma del ingreso correspondiente a todas las fuentes.

(12) La ecuación (1) viene dada por la convexidad del índice de desigualdad, y con la restricción de que $\mu_{\mathrm{k}}$ sea mayor o igual que cero para todo k. La discusión del tema se presenta en Kolm (1976) y Shorrocks (1978). 
Esta regla única viene dada por:

$$
R_{v}: s_{k}=\operatorname{cov}\left(Y_{k}, Y\right) / \sigma^{2}(Y)
$$

que es la regla de descomposición natural (13) para la varianza y el coeficiente de variación y que no depende del índice de desigualdad utilizado en el análisis.

Adicionalmente se presenta la descomposición del Gini. Utilizando la fórmula:

$$
G(Y)=\frac{2}{n^{2} \mu} \sum_{i}\left(i-\frac{n+1}{2}\right) Y_{i}
$$

las contribuciones serán:

$$
S_{k}=\frac{\mu_{k}}{\mu} \tilde{G}\left(Y_{k}\right)
$$

donde $\widetilde{G}(Y$ es el Pseudo - Gini (14) para los ingresos provenientes del factor k. Y las contribuciones proporcionales del factor $\mathrm{k}$

$$
R_{G}: s_{k}=\frac{\sum_{i}(i-(n+1) / 2) Y_{i k}}{\sum_{i}(i-(n+1) / 2) Y_{k}}
$$

\section{RESULTADOS}

Antes de presentar los resultados de las diferentes descomposiciones, se muestra algunas medidas de desigualdad para los años 1995 y 1998 en el cuadro 1 . Aunque todos ellos se incrementan, la variación en algunos es más significativa que en otros. El aumento en el coeficiente de Gini, índice sensible a cambios en la parte media de la distribución, es menos importante que el incremento en la razón de quintiles, índice sensible a variaciones en las colas de la distribución. Es decir, la evidencia sugiere que en el período previo a la crisis se observó un incremento en la desigualdad mayormente explicado por variaciones en los extremos de la distribución y no en los segmentos medios de la misma. el índice.

(13) Las reglas de descomposición son naturales cuando surgen de la forma usual de escribir

(14) Antes de calcular el Gini, se ordena los ingresos de menor a mayor, ya que este ordenamiento influye en las ponderaciones asignadas a cada hogar. El cálculo del pseudo-Gini para cada fuente, no se realiza ordenando los ingresos de esa fuente, sino sobre el mismo orden de los ingresos totales del hogar. Por esto es un pseudo-Gini, y no un Gini. 


\section{Cuadro 1 - Índices de Desigualdad para el Ingreso per Cápita - Ecuador.}

\begin{tabular}{|c|c|c|}
\hline Índice & 1995 & 1998 \\
\hline Q1 & 1,82 & 1,49 \\
Q2 & 6,69 & 6,35 \\
Q3 & 11,74 & 11,43 \\
Q4 & 19,98 & 19,81 \\
Q5 & 60,07 & 60,92 \\
Q5/Q1 & 33,01 & 40,89 \\
Varianza del Log. Ingreso & 1,493 & 1,621 \\
Gini & 0,572 & 0,580 \\
Coeficiente de Variación & 1,579 & 1,788 \\
\hline
\end{tabular}

Para indagar cuáles habrían sido los factores más importantes en explicar el nivel y los cambios en desigualdad, se utiliza la descomposición de Fields. Una vez determinados estos elementos claves, se realiza la descomposición de Shorrocks para analizar cómo al interior de cada grupo, las distintas fuentes de ingreso familiar contribuyen a la desigualdad.

\section{1. Descomposición de Fields}

La descomposición de Fields utiliza un modelo de ingresos, estimado por la metodología de Heckman en dos etapas. La ecuación de ingresos es:

$\ln \mathrm{Y}=\beta_{0}+\beta_{1} *$ escolaridad $+\beta_{2} *$ experiencia $+\beta_{3} *$ experiencia ${ }^{2}+\beta_{4} *$ género

$+\beta_{5} *$ etnia $+\beta_{6} *$ informalidad $+\beta_{7} *$ sector público $+\beta_{8} *$ sector agrícola

$+\beta_{9} *$ escolaridad padres $+\sum_{i} \beta_{\mathrm{i}} *$ rama de actividad 
Las estimaciones para los años 1995 y 1998 son presentadas en el cuadro 2 (15).

Cuadro 2 - Resultados de las estimaciones de la ecuación de Ingresos 1995 y 1998 (Especificaciones corregidas por el sesgo de selección).

\begin{tabular}{|c|c|c|c|c|}
\hline Variables & 1995 a (16) & $1995 \mathrm{~b}$ & 1998 a & $1998 \mathrm{~b}$ \\
\hline Constante & $\begin{array}{c}12,064 \\
(138,49)\end{array}$ & $\begin{array}{c}11,879 \\
(122,77)\end{array}$ & $\begin{array}{c}12,625 \\
(118,21)\end{array}$ & $\begin{array}{l}12,328 \\
(108,1)\end{array}$ \\
\hline Años de escolaridad & $\begin{array}{c}0,081 \\
(21,95)\end{array}$ & $\begin{array}{c}0,082 \\
(21.75)\end{array}$ & $\begin{array}{l}0,079 \\
(20.2)\end{array}$ & $\begin{array}{c}0,08 \\
(20.22)\end{array}$ \\
\hline Experiencia potencial & $\begin{array}{c}0,03 \\
(6,88)\end{array}$ & $\begin{array}{l}0,029 \\
(6,62)\end{array}$ & $\begin{array}{l}0,036 \\
(7,08)\end{array}$ & $\begin{array}{l}0,037 \\
(7,42)\end{array}$ \\
\hline$(\text { Experiencia potencial })^{2}$ & $\begin{array}{c}-0,00044 \\
(-5,71)\end{array}$ & $\begin{array}{c}-0,00042 \\
(-5,53)\end{array}$ & $\begin{array}{c}-0,00056 \\
(-6,65)\end{array}$ & $\begin{array}{c}-0,00058 \\
(-6,94)\end{array}$ \\
\hline Mujer $=1$ & $\begin{array}{c}-0,566 \\
(-19,71)\end{array}$ & $\begin{array}{c}-0,504 \\
(-16,69)\end{array}$ & $\begin{array}{c}-0,536 \\
(-16,95)\end{array}$ & $\begin{array}{r}-0,472 \\
(-14,37)\end{array}$ \\
\hline Origen étnico $=1$ & $\begin{array}{c}-0,282 \\
(-, 36)\end{array}$ & $\begin{array}{c}-0,275 \\
(-3,6)\end{array}$ & $\begin{array}{c}-0,289 \\
(-4,1)\end{array}$ & $\begin{array}{l}-0,274 \\
(-3,89)\end{array}$ \\
\hline Padre Universitario $=1$ & $\begin{array}{l}0,391 \\
(5,23)\end{array}$ & $\begin{array}{l}0,371 \\
(5,01)\end{array}$ & $\begin{array}{l}0,404 \\
(6,22)\end{array}$ & $\begin{array}{l}0,379 \\
(5,96)\end{array}$ \\
\hline Madre Universitaria $=1$ & $\begin{array}{l}0,033^{*} \\
(0,29)\end{array}$ & $\begin{array}{l}0,036^{*} \\
(0,33)\end{array}$ & $\begin{array}{l}0,337 \\
(2,98)\end{array}$ & $\begin{array}{l}0,353 \\
(3,07)\end{array}$ \\
\hline Trabajador Agrícola $=1$ & $\begin{array}{c}-0,643 \\
(-19,16)\end{array}$ & $\begin{array}{c}-0,467 \\
(-11,14)\end{array}$ & $\begin{array}{c}-0,614 \\
(-18,93)\end{array}$ & $\begin{array}{l}-0,376 \\
(-8,35)\end{array}$ \\
\hline Sector Público = 1 & $\begin{array}{l}0,049 \\
(1,59)\end{array}$ & $\begin{array}{l}0,088 \\
(2,61)\end{array}$ & $\begin{array}{l}0,195 \\
(6,24)\end{array}$ & $\begin{array}{l}0,269 \\
(7,85)\end{array}$ \\
\hline Sector Informal = 1 & $\begin{array}{c}-0,374 \\
(-16,39)\end{array}$ & $\begin{array}{c}-0,381 \\
(-16,51)\end{array}$ & $\begin{array}{l}-0,394 \\
(-16,5)\end{array}$ & $\begin{array}{c}-0,405 \\
(-16,43)\end{array}$ \\
\hline Manufactura (17) & & $\begin{array}{c}0,044 * \\
(0,61)\end{array}$ & & $\begin{array}{l}0,184 \\
(2,39)\end{array}$ \\
\hline
\end{tabular}

(15) Dichas estimaciones fueron realizadas por el método de Heckman Full Information para corregir el problema de sesgo de selección. El modelo que explica la decisión de participación utiliza como variables explicativas la escolaridad, una dummy si el individuo es casado, una dummy si es jefe de hogar y una dummy si tiene hijos menores de 5 años. incluye.

(16) La especificación (a) excluye las ramas de actividad económica. La especificación (b) las

(17) Se excluyó la rama de agricultura. 


\begin{tabular}{|c|c|c|c|c|}
\hline Minería & & $\begin{array}{c}0,049 * \\
(0,96)\end{array}$ & & $\begin{array}{l}0,241 \\
(4,16)\end{array}$ \\
\hline Electricidad & & $\begin{array}{l}0,304 \\
(7,69)\end{array}$ & & $\begin{array}{l}0,321 \\
(6,72)\end{array}$ \\
\hline Construcción & & $\begin{array}{l}0,253 \\
(6,84)\end{array}$ & & $\begin{array}{l}0,263 \\
(6,53)\end{array}$ \\
\hline Comercio & & $\begin{array}{l}0,436 \\
(9,16)\end{array}$ & & $\begin{array}{c}0,568 \\
(11,62)\end{array}$ \\
\hline Transporte & & $\begin{array}{l}0,191 \\
(3,81)\end{array}$ & & $\begin{array}{l}0,249 \\
(5,55)\end{array}$ \\
\hline Servicios varios & & $\begin{array}{l}0,01 * \\
(0,24)\end{array}$ & & $\begin{array}{l}0,168 \\
(3,57)\end{array}$ \\
\hline Wald chi ${ }^{2}$ & 2468,15 & 2190,31 & 2670,42 & 2958,58 \\
\hline Número de Observaciones & 16839 & 16839 & 15951 & 15951 \\
\hline
\end{tabular}

(t) Estadístico t se muestra entre paréntesis * Coeficiente estadísticamente no significativo

El retorno de la educación se ubica en torno al 8\%, rango compatible con la evidencia empírica existente en el tema (Samaniego, 1995). Este coeficiente es altamente significativo y como se mostrará posteriormente también tiene poder explicativo en la dispersión de los ingresos.

La contribución de un año adicional de experiencia laboral (potencial) se encuentra entre el $3 \%$ y el $4 \%$. El signo del coeficiente asociado al término cuadrático de experiencia es negativo, coincidente con patrones de ingreso y edad. La variable género (mujer $=1$ ), tiene un coeficiente negativo, lo que sería evidencia clara de discriminación por sexo, donde, controlando por capital humano y otras características del mercado de trabajo, una mujer gana alrededor del $50 \%$ menos que un hombre.

El pertenecer a un grupo étnico (18) también es causa de discriminación como se muestra en los resultados. Una persona de raza indígena gana cerca del $30 \%$ menos que una que no lo es. (19) La educación del padre y la madre es incluida en forma de

(18) La variable etnia es una dummy que toma el valor de 1 en el caso que la persona hable algún idioma indígena, como quechua o shuar. Es decir no está efectivamente medida, sino que es una aproximación, aunque algo incompleta, de la raza del individuo. Para corroborarlo basta con notar que el $4 \%$ de la muestra es de procedencia étnica, cuando se estima que en realidad alrededor del $25 \%$ de la población ecuatoriana es indígena.

(19) Contreras \& Galván (2002) encuentran evidencia similar para el caso boliviano, con un $24 \%$ de reducción en su ingreso. Su análisis se extiende incluso para el caso de mujeres de origen étnico, cuya condición es objeto de discriminación. 
dummy, (universitario = 1). Un padre con nivel de instrucción superior ejercería una influencia positiva en el ingreso del individuo. Mientras que la educación de la madre parece no tener importancia en el ingreso laboral en 1995. Lam \& Schoeni (1993) muestran este efecto intergeneracional en Brasil y concluyen que la educación de los padres se transmite a sus hijos en mayor escolaridad e ingresos.

En el caso de trabajadores del sector público, se encuentra un retorno positivo en el ingreso del individuo. Este retorno podría ser mayor en alrededor del 15\% en 1998. Trabajar en una empresa pública representaría beneficios adicionales en el ingreso, que los trabajadores del sector privado no están percibiendo.

El grado de formalidad de la firma (20) también parece ser importante para determinar el nivel de ingresos de un individuo. Los trabajadores del sector informal ganan menos que los trabajadores del sector formal, resultado congruente con la evidencia. Como se indicó en la sección II, el sector informal se ha visto acrecentado con la crisis, aun cuando los ingresos serían inferiores con respecto al sector moderno.

Se incluye una categoría adicional en las estimaciones: ramas de actividad económica de la firma. Trabajar en cualquiera de las actividades resulta un pago más alto que el trabajar en la rama excluida (agricultura), como lo indican los coeficientes asociados a estas variables. Este hecho hace evidente las diferencias salariales de los trabajadores de firmas del sector agrícola.

Este elemento no ha sido analizado en detalle, a pesar de la importancia de un sector económico tradicional en la economía ecuatoriana, principalmente en producción para la exportación. La posición ocupacional sirve como evidencia adicional para validar esta hipótesis. Los trabajadores del sector agrícola perciben ingresos inferiores en alrededor del $50 \%$.

Toda la evidencia respalda el análisis posterior. Los resultados se usan para medir las contribuciones a la desigualdad de cada variable incluida en la regresión, de acuerdo a la metodología desarrollada en la sección 2. 1. Los resultados se presentan en el cuadro 3.

De los factores incluidos, la educación contribuye en mayor grado a explicar la varianza del logaritmo del ingreso. En 1995 explica un 44\% de la desigualdad, medida que se reduce levemente en 1998. Otro factor que explica la desigualdad es la informalidad de la firma, cerca del $15 \%$ en ambos períodos.

La descomposición de Fields sugiere cambios en la contribución de dos variables. En primer lugar la experiencia (21), que aun con una contribución pequeña, sufre un incremento en el período de análisis, de $2 \%$ en 1995 a $7 \%$ en 1998. Por otra parte, el cambio más importante en contribuciones a la desigualdad lo sufre la variable sector agrícola que reduce su participación en 7 puntos porcentuales.

(20) Esta variable tampoco está medida efectivamente. Se utiliza como proxy el tamaño de la firma. El supuesto es que firmas con cinco empleados o menos pertenecerían al sector informal.

(21) Es importante destacar que la experiencia es incluida en la ecuación de ingresos con un término lineal y un término cuadrático y su contribución total se computa agregando ambas contribuciones. 


\section{Cuadro 3 - Contribuciones porcentuales de la varianza del logaritmo del ingreso explicada, computadas por la Metodología de Fields 1995 y 1998.}

\begin{tabular}{|c|c|c|c|}
\hline Variables & $\mathbf{1 9 9 5}$ & $\mathbf{1 9 9 8}$ & Variación \\
\hline Educación & 43,72 & 41,13 & $-2,59$ \\
Experiencia & 2,23 & 7,27 & 5,04 \\
Género & 12,88 & 10,00 & $-2,88$ \\
Etnia & 1,30 & 1,55 & 0,25 \\
Educación padres & 1,98 & 3,04 & 1,06 \\
Sector público & 1,24 & 2,57 & 1,33 \\
Sector agrícola & 19,36 & 12,06 & $-7,30$ \\
Informalidad & 14,25 & 15,04 & 0,79 \\
Total ramas de actividad & 3,04 & 7,34 & 4,30 \\
\hline
\end{tabular}

En principio, como preámbulo de la crisis se habría generado creciente desempleo de manera que solamente los trabajadores más aptos, se habrían mantenido en sus puestos de trabajo y de aquellos que rotaron, los que presentaron mayor experiencia se habrían ubicado en empleos más ventajosos. De esta forma, el tener experiencia previa, se habría constituido en una fuente de inequidad.

Con respecto a la pertenencia al sector agrícola, se habría reducido la importancia de este factor al generar desigualdad. Para explicar esta variación, hay que considerar algunos criterios. En primer lugar, entre 1997 y 1998 el fenómeno del Niño afectó duramente a la economía ecuatoriana. En el sector agrícola, las pérdidas fueron mayores que en los demás sectores y esta situación habría afectado el empleo y las decisiones de participación de los familiares del jefe agrícola. Un efecto podría haber sido la migración campo-ciudad que no ha perdido relevancia en el país. Por otra parte, la estructura productiva en el grupo agrícola presenta un alto porcentaje de trabajadores con empleo no remunerado (22).

Vale destacar que un análisis adicional de la descomposición de Fields puede dar luz al momento de explicar qué elementos importan en la contribución de estos factores. El cuadro 5a del Anexo presenta la importancia relativa de los tres criterios que explican la contribución: el retorno, la correlación del factor con el ingreso y la desviación estándar del factor.

(22) Las cifras que validan esto son tomadas del Censo Nacional Agropecuario (2000). La diferencia entre su análisis y el presente es que ellos definen una UPA (Unidad de Producción Agropecuaria) como una familia agrícola, mientras que aquí se define como agrícola al grupo cuyo jefe declara ser trabajador agrícola. El 16,25\% de los residentes ocupados de la UPA trabajan en otros sectores de actividad. Sólo el 3,27\% de los miembros de la UPA recibe una remuneración por su empleo y el $80,48 \%$ restante es empleado no remunerado. 
En el caso de la experiencia, el factor más importante son los retornos en ambos casos. En la pertenencia al sector agrícola, la composición es distinta puesto que el retorno de ser trabajador agrícola es tan importante como la correlación del factor con el ingreso.

Cabe destacar que existe un conjunto importante de factores que no es posible explicar tanto en el ingreso como en la desigualdad. La parte de varianza del logaritmo del ingreso explicada corresponde sólo al 40\%. A pesar de esta limitación, se habría identificado las características del individuo que contribuyen en mayor grado a la desigualdad. No habría existido un efecto importante o al menos inmediato en el período analizado sobre la proporción en que las características del individuo explican la desigualdad. La siguiente sección analiza en otro sentido las fuentes de inequidad.

\section{NO HAY CRISIS SIN CAMBIOS: ¿CUÁNTO IMPORTAN LAS DIFERENCIAS AL INTERIOR DE GRUPOS?}

La sección anterior sugiere que la desigualdad habría aumentado en el contexto previo a la crisis económica y política. Algunas características del individuo estarían explicando gran parte de la desigualdad. Aun cuando no se observan cambios en las contribuciones porcentuales, explorar más a fondo estas características puede ayudar a explicar los fenómenos a través de los cuales las crisis se traducen en desigualdad.

En esta sección se hace un análisis de la forma en que el género, la formalidad en el empleo, el nivel de escolaridad y la pertenencia al sector económico agrícola, características anteriormente identificadas, generan diferencias de ingresos entre los hogares. Con esta finalidad, la metodología de descomposición de Shorrocks es utilizada para descomponer la desigualdad al interior de estos grupos (23).

Los principales índices de desigualdad computados por grupo muestran de forma preliminar las diferencias existentes. El siguiente cuadro presenta los índices de cada grupo en 1995 y 1998.

Cuadro 4 - Índices de desigualdad por grupos.

\begin{tabular}{|c|cc|cc|cc|}
\hline \multirow{2}{*}{ Jefe de familia } & \multicolumn{2}{|c|}{ Gini } & \multicolumn{2}{c|}{$\begin{array}{c}\text { Coeficiente } \\
\text { de variación }\end{array}$} & \multicolumn{2}{c|}{$\begin{array}{c}\text { Varianza del log. } \\
\text { del ingreso }\end{array}$} \\
\hline \multirow{2}{*}{ Agrícolas } & 1995 & 1998 & 1995 & 1998 & 1995 & 1998 \\
\cline { 2 - 7 } Mujeres & 0,637 & 0,608 & 1,822 & 1,688 & 1,857 & 1,941 \\
Hombres & 0,643 & 0,619 & 1,837 & 1,740 & 1,902 & 2,050 \\
No Agrícolas & 0,634 & 0,597 & 1,825 & 1,589 & 1,826 & 1,863 \\
Mujeres & 0,522 & 0,535 & 1,440 & 1,656 & 0,977 & 1,087 \\
Hombres & 0,526 & 0,540 & 1,463 & 1,621 & 0,990 & 1,099 \\
& 0,512 & 0,528 & 1,402 & 1,684 & 0,910 & 1,045 \\
\hline
\end{tabular}

(23) En el cuadro 6a del Anexo se presentan los resultados de la descomposición de Shorrocks con la regla de la varianza, del Gini y las contribuciones porcentuales de cada fuente al ingreso familiar para la muestra completa. 


\begin{tabular}{|c|cc|cc|cc|}
\hline Jefe de familia & \multicolumn{2}{|c|}{ Gini } & \multicolumn{2}{c|}{$\begin{array}{c}\text { Coeficiente } \\
\text { de variación }\end{array}$} & \multicolumn{2}{c|}{ Varianza del log. } \\
del ingreso
\end{tabular}

El grupo agrícola (25) presenta mayores niveles de desigualdad que el grupo no agrícola en todos los índices. Esta observación es coincidente con estudios anteriores realizados en el tema para Ecuador. La capacidad en el sector agrícola para generar equidad se ve limitada por la concentración en la distribución de los factores básicos como tierra (26) y capital. Adicionalmente, la utilización del recurso humano para este sector no requiere mayor capacitación ni instrucción formal dando como resultado bajos niveles de ingresos. La relevancia del sector en economías como la ecuatoriana no es discutible por la alta dependencia de la producción en el sector primario.

El grupo de trabajadores formales sería más desigual en la distribución de su ingreso que el grupo informal según el coeficiente de Gini, aunque la varianza del logaritmo sugiere lo contrario. Es decir, la distribución de ingresos de los hogares en el grupo de empleo informal sería más homogénea en el centro de la distribución que en el grupo de empleo formal. Mientras que lo opuesto sucede en las colas de la distribución, donde existe mayor diferencia entre ricos y pobres en el grupo informal.

El grupo de familias separado por género del jefe muestra que en ambos casos la desigualdad aumentó en el tiempo. Cabe destacar que cuando el jefe es mujer, la desigualdad es mayor. Todos los índices mostrados sugieren iguales resultados.

La desigualdad es mayor en el grupo de familias con jefe no capacitado, aunque la desigualdad parece no cambiar en el período para este grupo. En el caso de familias con jefe capacitado, se observa un incremento en la desigualdad por causa de la crisis al interior del grupo.

(24) El criterio elegido para definir un trabajador capacitado es 5 años de escolaridad. Se eligió este valor debido a que el promedio de escolaridad es 5 años en 1995 y 6 años en 1998. De Jong \& Vos utilizan el mismo criterio para separar trabajadores capacitados y no capacitados.

(25) Cuyo jefe de familia es agrícola, no necesariamente todos los demás miembros de la familia lo son. Alrededor del $60 \%$ de los demás miembros del hogar cuyo jefe es agrícola también pertenecen a este sector.

(26) El coeficiente de Gini de distribución de la tierra en Ecuador es 0,80 en el año 2000. 
Los resultados anteriores darían elementos para comprender la medida en que afectó la crisis a estos grupos y a su vez cómo cada grupo contribuyó a la desigualdad observada. Sin embargo, las respuestas todavía son muy limitadas. La descomposición por fuentes de ingreso al interior de los grupos permite un análisis más exhaustivo de las fuentes de desigualdad.

\section{1. Sector agrícola (27)}

La descomposición de Fields mostró que pertenecer al sector agrícola explicaría en una medida no despreciable la desigualdad. A continuación, el interés se centra en examinar cómo cada fuente de ingreso familiar contribuye a explicar la desigualdad al interior de este grupo.

Cuadro 5 - Contribuciones a la Desigualdad del Grupo Agrícola.

\begin{tabular}{|l|rrr|rrr|}
\hline & \multicolumn{3}{|c|}{$\begin{array}{c}\text { Contribución a la } \\
\text { desigualdad }\end{array}$} & \multicolumn{3}{c|}{$\begin{array}{c}\text { Participación } \\
\text { en el ingreso }\end{array}$} \\
\hline Fuentes & $\mathbf{1 9 9 5}$ & $\mathbf{1 9 9 8}$ Variación & $\mathbf{1 9 9 5}$ & $\mathbf{1 9 9 8}$ Variación \\
\hline & \multicolumn{3}{|c|}{ Ingreso laboral } & & & \\
Jefe de familia & 19,11 & 19,58 & 0,47 & 25,23 & 28,89 & 3,36 \\
Cónyuge & 20,75 & 5,07 & $-15,68$ & 10,23 & 7,30 & $-2,93$ \\
Otros miembros & 41,09 & 54,94 & 13,85 & 38,51 & 43,68 & 5,17 \\
\hline & \multicolumn{3}{|r|}{ Ingreso no laboral } & & & \\
Ayuda familiar & 3,60 & 9,15 & 5,55 & 8,46 & 9,81 & 1,35 \\
Donaciones Instituciones & 0,07 & 0,07 & 0 & 0,51 & 0,12 & $-0,39$ \\
Impuestos & $-0,45$ & $-0,58$ & $-0,13$ & $-0,55$ & $-0,78$ & $-0,23$ \\
Pensiones & 3,48 & 7,35 & 3,87 & 5,72 & 6,73 & 1,01 \\
Rentas del capital & 9,82 & 3,92 & $-5,9$ & 8,57 & 3,62 & $-4,95$ \\
Otros ingresos & 2,53 & 0,49 & $-2,04$ & 3,01 & 0,63 & $-2,38$ \\
Total & 100,00 & 100,00 & 0,00 & 100,00 & 100,00 & 0,00 \\
\hline
\end{tabular}

El cuadro 5 muestra que la desigualdad cambió de fuentes explicativas en el período 1995-1998, principalmente en términos del ingreso laboral, donde se muestra una reducción en el grado de explicación del ingreso del cónyuge y un incremento en la contribución de los demás miembros del hogar. Estos cambios que operan en sentidos opuestos podrían haberse producido por dos razones. En primer lugar, la movilidad agrícola.

(27) $30 \%$ de los trabajadores en la muestra y $40 \%$ de los jefes de familia pertenecen al sector 
dentro de las familias de un sector a otro (28), agrícola a no agrícola y viceversa, y en segundo lugar por cambios en la tasa de participación en el trabajo.

Específicamente para el ingreso de otros miembros, la explicación se encontraría en que los hijos y otros familiares de este grupo estarían participando de manera más activa en el trabajo que en el caso de familias no agrícolas (29) y eventualmente realizando actividades productivas que les dieron remuneraciones con un efecto distributivo negativo. Las tasas de participación promedio de los miembros de hogares agrícolas son $39 \%$ y $43 \%$ en 1995 y 1998, respectivamente. En el caso de hogares no agrícolas, la tasa es $33 \%$ y $34 \%$ en los mismos períodos. Efectivamente los integrantes de hogares "agrícolas" participan más que en hogares "no agrícolas" (30).

Los cambios en la contribución del cónyuge se pueden explicar con variaciones en la participación femenina, ya que el $95 \%$ de los cónyuges del jefe son mujeres. Tal como se muestra en el cuadro 7a del anexo, la participación femenina por quintiles de ingreso en el sector agrícola crece en todos los quintiles y con mayor fuerza en los dos primeros. De estas cónyuges, el $57 \%$ también pertenecía al sector agrícola en 1995 y el $62 \%$ en 1998 . Es decir, la desigualdad aumentó porque la mujer cuyo esposo es agrícola participó más en el trabajo y también porque participó más dentro del mismo sector agrícola.

Otro hecho que destaca es la baja participación del ingreso laboral en el total con respecto al otro grupo. Un $75 \%$ del ingreso familiar proviene del trabajo, mientras que en el grupo no agrícola, esta porción es del 90\%. Un parte considerable de su ingreso, lo constituyen las ayudas de familiares y las rentas del capital. Existe también una diferencia considerable en las fuentes que generan mayor desigualdad. El ingreso del jefe de familia constituye una porción menor al $20 \%$ del ingreso en caso de familias agrícolas y más del $60 \%$ en el caso de familias no agrícolas. Finalmente, hay que considerar que la mayoría de las personas en el sector trabajan sin remuneración, como se anotó anteriormente, lo cual no permite capturar todo el efecto en este grupo.

El cuadro 8a del Anexo presenta las contribuciones a la desigualdad del grupo de familias no agrícolas. De igual manera, cada fuente explica la desigualdad en forma distinta para este grupo. El ingreso del jefe explica en 1995 un 58\% de la desigualdad, cifra que en 1998 se incrementa marginalmente al 59\%. Por el contrario, el ingreso de la cónyuge mostraría cambios importantes, pasando a explicar del 14\% al 21\% entre 1995 y 1998.

(28) Este hecho se justifica en los efectos de la crisis sobre la estructura del empleo, que se traduce en desigualdad, cuya existencia es mostrada por De Jong y Vos. Adicionalmente, Martínez (1999) postula que se han dado cambios importantes en la forma de participación de la mujer que reside en zonas rurales, de trabajar en el sector agrícola a otro tipo de actividades como textiles, artesanales y comerciales.

(29) Familias cuyo jefe pertenece al sector agrícola tendrían una estructura diferente, dados los bajos ingresos del jefe, el número de miembros de la familia que debe recurrir al trabajo es mayor, por eso la contribución de la fuente otros miembros es más importante. Efectivamente el ingreso per capita de los trabajadores agrícolas en 1995 corresponde al $49 \%$ de los ingresos de los trabajadores no agrícolas, porción que en 1998 se reduce al 42\%. Esto estaría implicando diferencias en la desigualdad entre grupos generadas por la diferencia en la estructura de las familias.

(30) Esto a pesar de que las tasas de participación de individuos que trabajan en el sector agrícola es inferior a la tasa de participación de individuos en el sector no agrícola. En 1995 la tasa de participación en este sector fue 33\% y en 1998 ascendió a 35\%. 
La Figura 1 muestra la profundización en las diferencias entre los grupos agrícola y no agrícola. Incluso, el efecto en pobreza, parece ser mayor que en desigualdad, debido al aumento de la distancia relativa entre las medias de ingresos de familias de uno y otro grupo.

1995

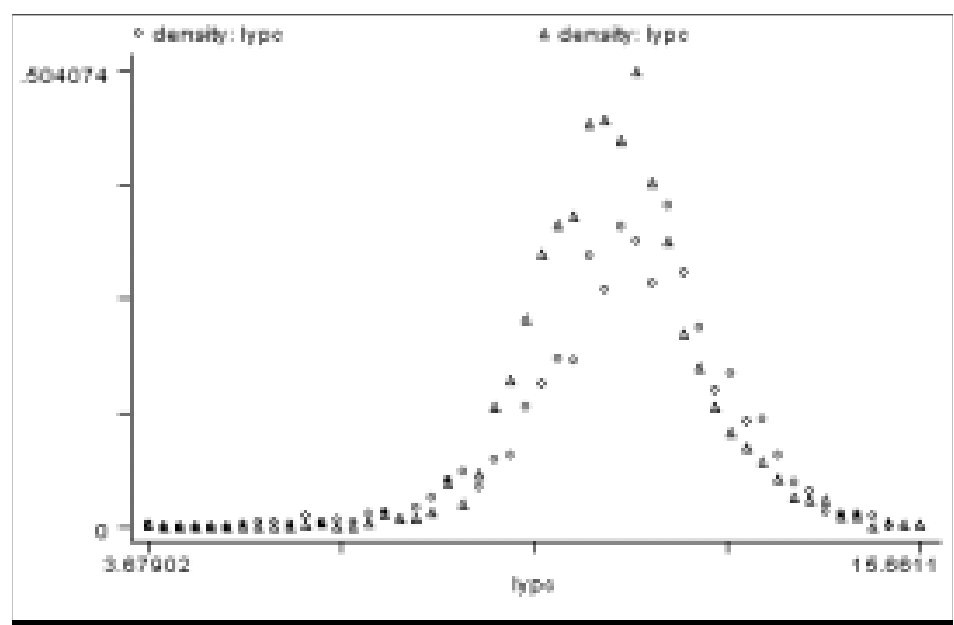

1998

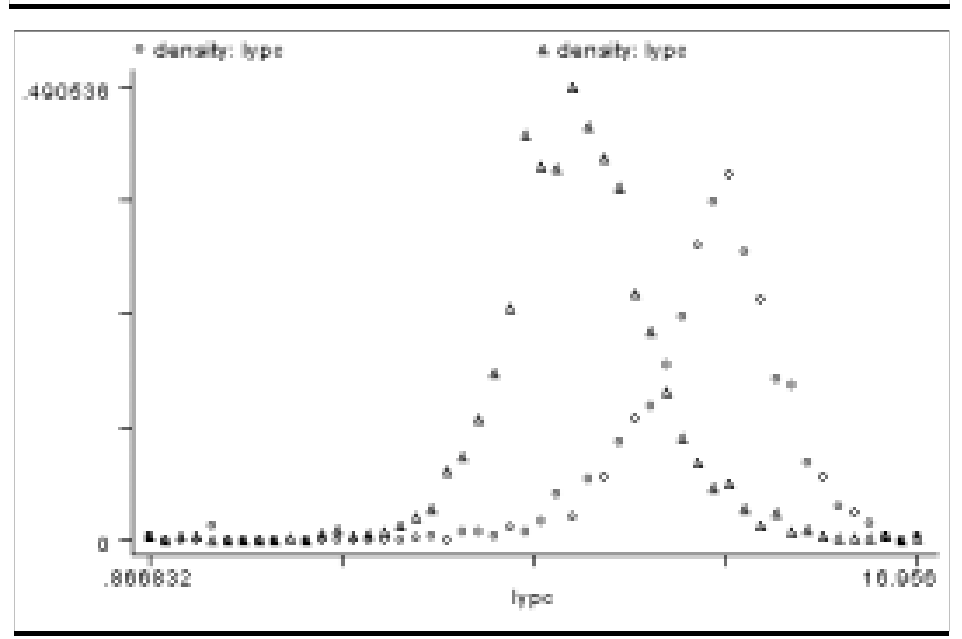

$\Delta$ Familias del Grupo Agrícola

o Familias del Grupo No Agrícola

Fig. 1 - Distribución de Ingresos por pertenencia al sector agrícola del jefe.

Los cambios que se reflejan en el ingreso del cónyuge, varían en distinta forma en el grupo agrícola y el no agrícola, pero en ambos casos son significativos. Esto podría ser un tema de la discutida evidencia del efecto de la participación de la mujer en los niveles de desigualdad que se analiza a continuación desde otra perspectiva. 


\section{2. Género}

El género es una característica clave en el análisis de la desigualdad. El ingreso laboral del hombre sería en promedio más alto que el de la mujer y esto contribuye a generar mayor desigualdad. En este caso, se mostrará cómo el género del jefe de hogar puede hacer diferencia al momento de explicar la desigualdad por fuentes de ingreso, es decir como un hogar es económicamente distinto de otro sólo porque el jefe es hombre o mujer.

El ingreso de familias cuyo jefe es mujer se descompone para explicar la desigualdad. La estructura del ingreso familiar es distinta a la observada en la muestra completa. El jefe de familia aporta con cerca del $30 \%$ del ingreso y los otros miembros del hogar lo hacen en una proporción similar, tal como se muestra en el cuadro 6.

Los cambios observados están concentrados en dos fuentes específicas. La primera es el ingreso laboral del jefe cuya contribución se reduce en el período analizado en $11 \%$. Este cambio sugeriría una disminución en la desigualdad de ingresos de las mujeres que son jefes de familia.

En 1995, el Gini para este grupo fue 0,78 y en 1998 se redujo a 0,76. La variación podría parecer poco importante e indicar que debiera existir otro mecanismo a través del

Cuadro 6 - Contribuciones a la Desigualdad de Grupo Jefe de Familia Mujer (31).

\begin{tabular}{|l|rrr|rrr|}
\hline & \multicolumn{3}{|c|}{$\begin{array}{c}\text { Contribución a la } \\
\text { desigualdad }\end{array}$} & \multicolumn{3}{c|}{$\begin{array}{c}\text { Participación } \\
\text { en el ingreso }\end{array}$} \\
\hline Fuentes & $\mathbf{1 9 9 5}$ & $\mathbf{1 9 9 8}$ & Variación & $\mathbf{1 9 9 5}$ & $\mathbf{1 9 9 8}$ & Variación \\
\hline \multirow{3}{*}{ Jefe de familia } & \multicolumn{3}{|c|}{ Ingreso laboral } & & & \\
Cónyuge & 39,26 & 28,56 & $-10,7$ & 33,87 & 32,91 & $-0,96$ \\
Otros miembros & 9,26 & 9,24 & $-0,02$ & 4,50 & 4,77 & 0,27 \\
& 29,52 & 29,91 & 0,39 & 35,89 & 35,86 & 0,03 \\
\hline \multirow{3}{*}{ Ayuda familiar } & \multicolumn{2}{|c|}{ Ingreso no laboral } & & & \\
Donaciones Instituciones & 6,13 & 25,91 & 19,78 & 12,24 & 18,53 & 6,29 \\
Impuestos & 0,01 & 0,04 & 0,03 & 0,34 & 0,06 & $-0,28$ \\
Pensiones & $-0,40$ & $-0,58$ & $-0,18$ & $-0,33$ & $-0,97$ & $-0,64$ \\
Rentas del capital & 1,16 & 3,76 & 2,6 & 4,07 & 4,39 & 0,32 \\
Otros ingresos & 7,01 & 2,84 & $-4,17$ & 6,92 & 3,94 & $-2,98$ \\
Total & 8,05 & 0,32 & $-7,73$ & 2,50 & 0,51 & $-1,99$ \\
& 100 & 100 & 0 & 100 & 100 & 0 \\
\hline
\end{tabular}

(31) Este grupo corresponde aproximadamente al 20\% del total de la muestra. 
cual los ingresos de estas mujeres se hicieron más homogéneos en tres años. Posiblemente estos cambios tengan relación con el aumento en las tasas de participación femenina, ya que producto de la crisis la mujer habría tenido que acudir al mercado de trabajo. Entre 1995 y 1998, la tasa de participación global aumentó en $1 \%$ y la tasa de participación femenina en $2 \%$ como se muestra en el cuadro 9a del anexo.

La ayuda familiar es otra fuente que muestra cambios importantes, al pasar de explicar la desigualdad en 6\% en 1995 a explicar 26\% en 1998. En hogares cuyo jefe es mujer, este componente del ingreso no laboral sería la tercera fuente en orden de importancia. Las variaciones podrían ser efecto de la migración de ecuatorianos al extranjero causada por la crisis y que empezó a crecer con mayor fuerza a partir de 1998. Como se ha observado a nivel agregado (32), las remesas del exterior se habrían convertido en un importante componente en ciertos hogares. Aunque en principio se estarían yendo tanto miembros de familias cuyo jefe es hombre como de aquellos con jefe mujer, en este último grupo el efecto es más visible y más importante.

Los hombres han sido los que tradicionalmente han dejado sus hogares para desplazarse a países como Estados Unidos en busca de un trabajo mejor remunerado y de esta forma su aporte al hogar se refleja como ayuda familiar y no como un ingreso laboral. Sin embargo estadísticas recientes muestran que precisamente después de 1995 las que más se han ido son las cónyuges y más que ellas, los hijos (33). Antes de 1995 el $60 \%$ de los migrantes eran hijos del jefe de hogar, proporción que se reduce al $49 \%$ en la segunda mitad de la década. La proporción de cónyuges que se habrían ido, creció de $5 \%$ a $11 \%$ entre estos mismos períodos, mientras que el $6 \%$ correspondiente a los jefes que se habría ido antes de 1995 se habría incrementado a $8 \%$.

Otra evidencia que sugiere el mismo resultado es que no ha variado la proporción de mujeres que siendo jefes de familia tienen cónyuge, la cual incluso se incrementó en un punto porcentual entre 1995 y 1998 . El caso de los hombres es diferente, del $88 \%$ de hombres que declaraban tener cónyuge en 1995, sólo un 83\% lo hace en 1998.

La fuente de desigualdad se encontraría en que alrededor del $80 \%$ de los hogares donde uno o más de sus miembros migraron pertenecen a los tres quintiles de ingresos más ricos de la población. Por lo tanto, en hogares donde el jefe es mujer, la ayuda familiar ha sido una fuente de inequidad al interior del grupo por causa de la migración, fenómeno que prosperó con la crisis. Efectivamente, las estructuras del hogar y los ingresos son distintas al utilizar el género del jefe para separar el análisis (34).

(32) Según BCE, en 1999 se recibió como remesas del extranjero cerca de 1100 millones de dólares. Esta cifra en 2000 fue de 1300 millones y en 2001 cercana a 1500 millones de dólares, alrededor del $60 \%$ de los ingresos petroleros, principal componente de los ingresos del país. Aunque estas son cifras oficiales, muchos autores consideran que subestiman las reales.

(33) Fuente: SIISE a partir de INEC, Encuesta de Medición de Indicadores de la Niñez y el Hogar (2000). La Encuesta Condiciones de Vida no tiene información sobre migración internacional de miembros del hogar. La sección migración solamente indaga sobre cambios en el lugar de residencia dentro del país.

(34) En el caso de familias cuyo jefe es hombre, la descomposición de Shorrocks muestra resultados muy distintos. Los cambios durante el período no revelan diferencias sustanciales. Destaca la importancia del ingreso del trabajo del jefe que aporta con más del $60 \%$ de los ingresos totales y explica el 54\% de la desigualdad. Para mayor detalle, véase el Cuadro 12a en el Anexo. 


\section{3. Escolaridad}

La escolaridad hace la contribución más importante a la desigualdad entre todas las variables de la descomposición de Fields. Conocer el efecto de la crisis en relación con esta variable es posible desagregando la muestra en grupos por rango de escolaridad.

Como se mostró anteriormente, el grupo no capacitado exhibe mayores niveles de desigualdad que el grupo capacitado, y aun cuando la diferencia es marginal, el factor educación habría contribuido a incrementar los niveles de desigualdad agregada, ya que también al interior de las familias se muestran incrementos. Los siguientes gráficos muestran la distribución de ingresos en ambos períodos para los dos grupos.

1995

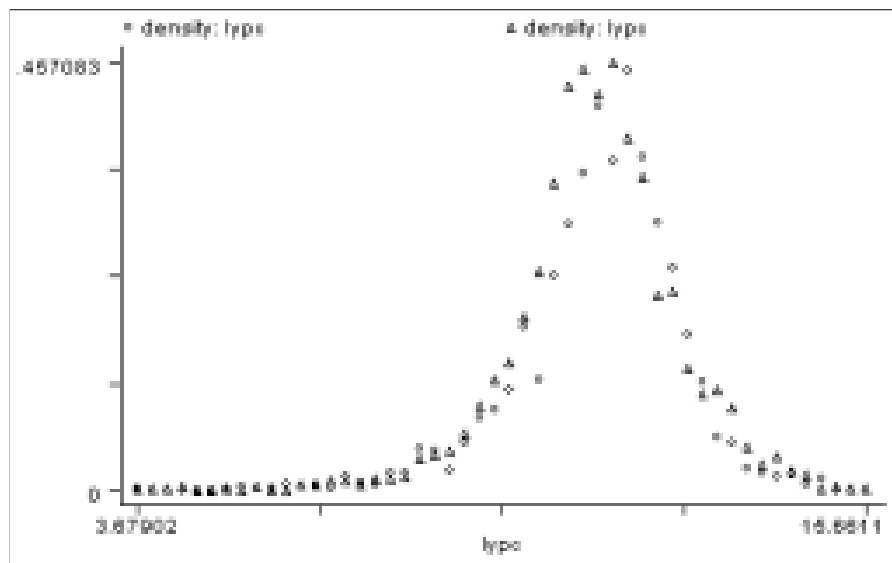

1998

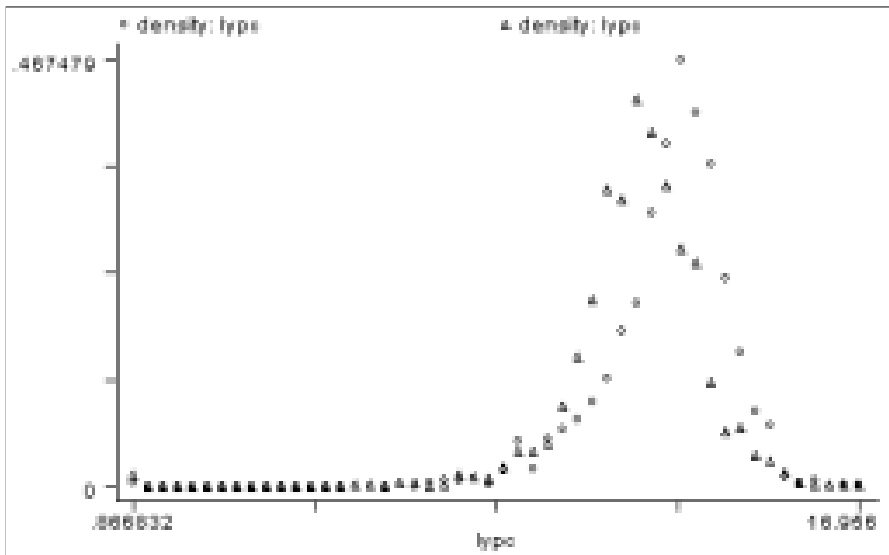

$\Delta$ Familias del Grupo No Capacitado

o Familias del Grupo Capacitado

Fig. 2 - Distribución de Ingresos por nivel de capacitación del jefe. 
Se presentan cambios entre un período y otro en la relación entre las distribuciones que podrían ser evidencia para mostrar incrementos en niveles de pobreza y desigualdad, y las diferencias explicadas por distintos niveles de educación.

El grupo de trabajadores capacitados corresponde al 62\% de la muestra en 1995 y al 66\% de la misma en 1998. El cuadro muestra las contribuciones para el grupo de trabajadores no capacitados.

Cuadro 7 - Contribuciones a la Desigualdad de Grupo - Jefe No Capacitado.

\begin{tabular}{|l|rrr|rrr|}
\hline & \multicolumn{3}{|c|}{$\begin{array}{c}\text { Contribución a la } \\
\text { desigualdad }\end{array}$} & \multicolumn{3}{c|}{$\begin{array}{c}\text { Participación } \\
\text { en el ingreso }\end{array}$} \\
\hline Fuentes & $\mathbf{1 9 9 5}$ & $\mathbf{1 9 9 8}$ & Variación & $\mathbf{1 9 9 5}$ & $\mathbf{1 9 9 8}$ & Variación \\
\hline & \multicolumn{3}{|c|}{ Ingreso laboral } & & & \\
Jefe de familia & 39,40 & 35,54 & $-3,86$ & 43,76 & 43,85 & $-0,09$ \\
Cónyuge & 11,90 & 3,78 & $-8,12$ & 8,91 & 5,73 & $-3,48$ \\
Otros miembros & 32,03 & 50,34 & 18,31 & 35,75 & 41,75 & 5,79 \\
\hline & \multicolumn{3}{|r|}{ Ingreso no laboral } & & & \\
Ayuda familiar & 2,38 & 4,98 & 2,6 & 4,86 & 6,20 & 1,34 \\
Donaciones Instituciones & $-0,005$ & 0,08 & 0,085 & 0,21 & 0,07 & $-0,14$ \\
Impuestos & $-0,15$ & $-0,09$ & $-0,06$ & $-0,23$ & $-0,36$ & $-0,13$ \\
Pensiones & 1,65 & 1,08 & $-10,37$ & 1,55 & 1,42 & $-0,13$ \\
Rentas del capital & 12,43 & 2,06 & $-10,37$ & 4,12 & 1,56 & $-2,56$ \\
Otros ingresos & 1,36 & 0,24 & $-1,12$ & 1,08 & 0,28 & $-0,8$ \\
Total & 100 & 100 & 0 & 100 & 100 & 0 \\
\hline
\end{tabular}

En familias con jefe de baja capacitación, el ingreso del jefe contribuye a explicar la desigualdad en una proporción que se reduce durante la crisis, tal vez por el bajo nivel de ingresos de estos individuos, lo cual obligó a una mayor participación de su grupo familiar. La mayor participación de cónyuges sería una menor fuente de inequidad en el tiempo. Por el contrario, el ingreso de otros miembros estaría explicando en mayor medida la desigualdad entre estas familias, al crecer en 18 puntos porcentuales.

El cuadro 11a del Anexo muestra las contribuciones para el grupo de jefes capacitados. La proporción en que las fuentes explican la desigualdad es muy distinta para este grupo. El jefe explica una mayor parte de la desigualdad y el cónyuge, aun cuando reduce su participación en el ingreso del hogar, estaría generando mayor desigualdad durante el período 1995-1998.

Por lo tanto, el nivel educativo del jefe de hogar se traduciría en otro tipo de factores al generar diferencias en desigualdad. Según De Jong \& Vos, esto se explica porque el grupo de trabajadores no calificados fue desplazado hacia el sector informal de la economía a la vez que mejoraron las condiciones de empleo e ingresos de trabajadores 
más calificados. Este razonamiento estaría exagerandoel efecto. Efectivamente, la escolaridad promedio de jefes de hogar pertenecientes al sector formal es cercana a 8 años superior al grupo de jefe del sector informal cuyo promedio de escolaridad es 6 años. Sin embargo, tanto en el grupo informal como en el formal, la escolaridad aumentó (35), evidencia que no valida el razonamiento anterior. El análisis de los hogares según el grado de formalidad en el empleo podría presentar evidencia más firme en el tema.

\section{4. Formalidad en el empleo}

La muestra se separa en dos grupos en función del grado de formalidad del empleo del jefe. El cuadro presenta los resultados de las contribuciones a la desigualdad de familias cuyo jefe labora en condiciones informales.

El ingreso del jefe de familia reduce su contribución en un $5 \%$ en el período analizado. En el caso de jefes informales la contribución de esta fuente a la desigualdad es mayor que en el caso de jefes formales. En el tiempo, se habría producido un desplazamiento de trabajadores del sector formal al sector informal de la economía producto de la crisis, de manera que trabajadores que eran formales en 1995, se habrían convertido en informales en 1998 (36). Según Jácome et al. (1998) hay una mayor segmentación del mercado laboral; con la reforma aumentó la brecha de salarios entre el sector moderno e informal y aumentó la demanda de trabajo calificado respecto al no calificado.

Cuadro 8 - Contribuciones a la Desigualdad del Grupo Empleo Informal.

\begin{tabular}{|l|rrr|rrr|}
\hline & \multicolumn{3}{|c|}{$\begin{array}{c}\text { Contribución a la } \\
\text { desigualdad }\end{array}$} & \multicolumn{3}{c|}{$\begin{array}{c}\text { Participación } \\
\text { en el ingreso }\end{array}$} \\
\hline Fuentes & $\mathbf{1 9 9 5}$ & $\mathbf{1 9 9 8}$ & Variación & $\mathbf{1 9 9 5}$ & $\mathbf{1 9 9 8}$ & Variación \\
\hline & \multicolumn{3}{|c|}{ Ingreso laboral } & & & \\
Jefe de familia & 56,51 & 51,78 & $-4,73$ & 57,55 & 59,45 & 1,9 \\
Cónyuge & 15,04 & 14,59 & $-0,45$ & 12,47 & 9,88 & $-2,59$ \\
Otros miembros & 18,73 & 14,36 & $-4,37$ & 20,81 & 20,46 & $-0,35$ \\
& \multicolumn{7}{|c}{ Ingreso no laboral } & & & \\
Ayuda familiar & 2,04 & 8,87 & 6,83 & 3,44 & 5,34 & 1,9 \\
Donaciones Instituciones & 0,02 & 0,12 & 0,1 & 0,07 & 0,07 & 0 \\
Impuestos & $-0,48$ & $-0,18$ & 0,3 & $-0,44$ & $-0,62$ & $-0,18$ \\
Pensiones & 0,43 & 1,11 & 0,68 & 1,41 & 1,54 & 0,13 \\
Rentas del capital & 6,18 & 8,61 & 2,43 & 3,47 & 3,24 & $-0,23$ \\
Otros ingresos & 1,53 & 0,74 & $-0,79$ & 1,24 & 0,64 & $-0,6$ \\
Total & 100 & 100 & 0 & 100 & 100 & 0 \\
\hline
\end{tabular}

(35) En el grupo de trabajadores formales la escolaridad promedio en años es 7,95 y 8,34, en 1995 y 1998 respectivamente. En el grupo informal, la escolaridad es 5,71 y 5,98 años en los mismos períodos.

(36) En las estadísticas para la muestra esta hipótesis no se verifica. Es posible que la medida de informalidad usada sea incompleta, pues no se incorpora más elementos que el tamaño de la firma. Los otros autores, consideran dentro del grupo informal a los trabajadores por cuenta propia. 
Otro factor que no se considera en este análisis es la relación de desigualdad con los niveles de desempleo y subempleo. Al respecto, De Jong y Vos hacen un análisis con microsimulaciones que permite identificar los signos de estos efectos y algunos otros factores. Su conclusión es que la crisis implicó que la desigualdad aumentara debido al incremento en la demanda de empleo calificado y se redujera por el cambio en la estructura remunerativa, el aumento en la participación y el aumento en el desempleo. Al no haber cambios en la composición de los trabajadores — según el grado de formalidad - en el tiempo, es posible que el incremento en desempleo entre 1995 y 1998 (37) se haya dado en ambos grupos. Construir las relaciones entre estas variables y la desigualdad es relativamente complicado y estaría fuera del alcance de este trabajo.

Cabe destacar la importancia que empiezan a tomar las fuentes de ingresos no laborales, como la ayuda familiar, por el efecto de la migración, antes discutido. Por el contrario, en el grupo formalmente empleado el ingreso del jefe de hogar es el factor más importante en explicar los cambios en la desigualdad en el tiempo.

La contribución del cónyuge no deja de ser importante en este caso, aunque su tendencia varía. En familias del sector informal, el ingreso del cónyuge contribuye a explicar la desigualdad en alrededor del $15 \%$ en ambos períodos. En el sector formal, se observa que la explicación de esta fuente crece en $4 \%$. Una explicación a este efecto podría encontrarse en las condiciones de empleo del jefe de familia que se traducen directamente a la tasa de participación de la mujer (38). Las cónyuges de familias en el sector informal participan menos en el trabajo que aquellas del sector formal.

En conclusión, un factor común a los cuatro grupos en que se desagregó la muestra es alguna condición especial en el trabajo de la cónyuge. Su participación en la fuerza laboral y el aporte de su ingreso a la familia tiene implicancias importantes sobre la desigualdad y hacia esos puntos debería dirigirse la investigación posterior en el tema.

\section{CONCLUSIONES}

El caso de Ecuador destaca por el alto nivel de desigualdad observado y por la profunda crisis económica y política que ha atravesado los últimos años. Los indicadores de desigualdad muestran variaciones entre el período 1995-1998. La evidencia indica que los factores más significativos para explicar la desigualdad son la escolaridad, la pertenencia al sector agrícola, el género y el grado de formalidad del empleo. Al desagregar la muestra por algunas de estas características se observan cambios importantes.

La relevancia de estos sectores en Ecuador es indiscutible. Siendo el sector agrícola una de las bases en que se sustenta la economía ecuatoriana, existen ciertas caractéristicas en las familias pertenecientes a este sector, especialmente sus condiciones laborales, que no son tan evidentes a primera vista. Este trabajo muestra que los

(37) Ver cuadro 13a del Anexo.

(38) Cabe recalcar que el criterio de separación es el jefe y no necesariamente los demás miembros del hogar también pertenecen al mismo sector. 
individuos que laboran en ese sector perciben ingresos muy inferiores que los demás. Otro factor de importancia es la diferencia entre hombres y mujeres en el nivel de ingresos y la contribución que esta diferencia hace a la desigualdad. El rol de la mujer al participar en el mercado laboral, y de manera especial de la jefe de hogar y la cónyuge, tendría implicaciones sobre la desigualdad que todavía no se han podido explicar con precisión.

Dados estos grupos, no es posible generalizar resultados, pero se tiene la ventaja de identificar factores específicos que son claves para la explicación y para reducción de la desigualdad. Políticas que compensen a los grupos donde existe mayor desigualdad, dirigiendo recursos en educación con énfasis en la mujer, promoviendo el crecimiento (y por ende el empleo) de sectores económicos más modernos, podrían permitir iniciar la lucha contra la desigualdad utilizando relativamente pocos recursos.

Este es un espacio donde se ha trabajado de manera limitada en el caso ecuatoriano. En esta misma línea, sería interesante incorporar el efecto de los ingresos por remesas familiares del extranjero y de los programas gubernamentales de ayuda en los años posteriores a 1998, información que estaría disponible en un futuro.

Agradecimientos

Los autores agradecen a Joseph Ramos y Osvaldo Larrañaga por los comentarios a una versión preliminar y a los participantes de la Reunión Anual de la Asociación de Economía de América Latina y el Caribe (LACEA, Madrid 2002). Se agradece también la valiosa asistencia de Rosanna Huayamave. Cualquier error es entera responsabilidad de los autores.

\section{Referencias citadas}

ACOSTA, A. 2001 - Ecuador: ¿Un modelo para América Latina? Reflexiones a dos años de la dolarización. Globalización.com

CONTRERAS, D. \& GALVÁN, M. A., 2002 - ¿Ha disminuido la discriminación salarial por género y etnia en Bolivia? Evidencia del período 1994 - 1999. Depto. de Economía, Universidad de Chile y Unidad de Análisis de Políticas Sociales y Económicas (UDAPE, Bolivia). Ms

DE JONG, N. \& VOS, R., 2000 - Rising Inequality during economic liberalization and crises: macro or micro causes in Ecuador's Case?, Working paper, No 326. La Haya: ISS.

EGUEZ, A., 2001 - Las remesas de emigrantes en Ecuador tras la dolarización In: La Economía de Mercado, virtudes e inconvenientes http://www.eumed.net/cursecon/colaboraciones/ Eguez-remesas-A.htm

FIELDS, G., \& YOO, G., 2000 - Falling labor Income Inequality in Korea's Economic Growth: Patterns and Underlying Causes. Review of Income and Wealth, Series 46, No 2: 139-160.

JÁCOME, L., LARREA, C. \& VOS, R. 1998 - Políticas macroeconómicas, distribución y pobreza en el Ecuador. Corporación de Estudios para el Desarrollo, Documento de Trabajo.

KOLM, S.-CH., 1976 - Unequal Inequalities. Journal of Economic Theory, XIII: 82-111 
LAM, D. \& SCHOENI, R., 1993 - Effects of Family Background on Earnings and Returns to Schooling: Evidence From Brazil, Journal of Political Economy, 101(4): 213-243.

LEÓN, M., 2001 - Los indicadores sociales. La migración internacional reciente: algunos interrogantes. Revista Gestión. No 90: 46-48; Ecuador: SIISE.

MARTINEZ, L., 1999 - Actividades rurales no agrícolas en Ecuador, Faculdad Latinoamericana de Ciencias Sociales, FLASCO. Working paper.

SAMANIEGO, P., 1995 - El ingreso y la educación en el Ecuador: análisis por niveles de instrucción. Cuestiones Económicas, № 24: 135-136; Banco Central del Ecuador.

SHORROCKS, A.F., 1978 - Income Ineqaulity and Income Mobility. Journal of Economic Theory, XIX: 379-93. 


\section{ANEXO 1}

\section{TRATAMIENTO DE LOS DATOS}

Este trabajo utiliza información de individuos y hogares de la Encuesta Condiciones de Vida (Living Standards Measurement Survey), realizada en Ecuador en 1995 y 1998. La institución a cargo de la elaboración y desarrollo de la encuesta es el Instituto Nacional de Estadística y Censos del Ecuador (INEC), con apoyo técnico del Banco Mundial.

Para la descomposición de Fields se utiliza el ingreso mensual del último trabajo de la semana pasada declarado por hombres y mujeres entre 10 y 65 años, rango considerado en la población económicamente activa (PEA) en Ecuador.

Para la descomposición de Shorrocks se utiliza el ingreso familiar total. La definición de ingreso familiar para la descomposición es la siguiente:

Ingreso Familiar = Ingreso Laboral + Ingreso No Laboral, donde

Ingreso Laboral $=$ Ingreso Laboral del Jefe de Familia + Ingreso Laboral del Cónyuge + Ingreso Laboral de los demás Miembros del Hogar, e

Ingreso No Laboral = Ayuda en dinero de Familiares + Donaciones de Instituciones + Impuestos + Pensiones de la Seguridad Social + Rentas del Capital

Para las medidas de pobreza y desigualdad se utiliza el ingreso total per cápita, sin ajustar por el tamaño del hogar. Tampoco se efectuaron ajustes por subdeclaración de ingresos (1).

\section{ANEXO 2}

Cuadro 1a - Estadísticas de la Muestra.

\begin{tabular}{|l|c|c|}
\hline Variables & $\mathbf{1 9 9 5}$ & $\mathbf{1 9 9 8}$ \\
\hline Género & & \\
Mujer & 50,40 & 50,11 \\
Hombre & 49,60 & 49,89 \\
\hline
\end{tabular}

(1) Este factor podría tener un efecto importante sobre los resultados, especialmente en el sector agrícola donde se estima que el ingreso reportado está muy por debajo del ingreso real. El ajuste por subdeclaración de ingresos se realiza comparando los ingresos de las encuestas con las cuentas nacionales, bajo el supuesto que estas son más confiables, tienen mayor cobertura y se realizan en base a una comprobación uso - fuente. La CEPAL utiliza factores de ajuste para categorías de ingreso y grupos de la población. La encuesta Condiciones de Vida es la fuente más confiable existente al momento y no ha sido sujeta a correcciones por sub-declaración. Sin embargo, para la Encuesta de Empleo del INEC, según Jácome el ingreso laboral declarado está subestimado en cerca de $5 \%$ y el ingreso no laboral en cerca de un $80 \%$, proporción que no es despreciable. 


\begin{tabular}{|c|c|c|}
\hline $\begin{array}{l}\text { Estado civil } \\
\% \text { casado }\end{array}$ & 47,51 & 47,52 \\
\hline \multicolumn{3}{|l|}{ Género del jefe de familia } \\
\hline Mujer & 16,87 & 16,78 \\
\hline Hombre & 83,13 & 83,22 \\
\hline \multicolumn{3}{|l|}{ Etnia } \\
\hline$\%$ etnia & 3,09 & 2,86 \\
\hline \multicolumn{3}{|l|}{ Rama de actividad } \\
\hline Agricultura & 36,52 & 37,15 \\
\hline Minería & 4,02 & 3,95 \\
\hline manufactura & 1,94 & 2,06 \\
\hline electricidad & 5,70 & 6,16 \\
\hline Construcción & 27,05 & 27,41 \\
\hline Comercio & 4,80 & 5,27 \\
\hline Transporte & 4,08 & 5,27 \\
\hline Servicios financieros & 5,49 & 5,45 \\
\hline Servicios varios & 10,39 & 8,10 \\
\hline \multicolumn{3}{|l|}{ Posición laboral } \\
\hline Empleado público & 6,81 & 6,15 \\
\hline Empleado privado & 29,04 & 28,33 \\
\hline Cuenta propia & 23,37 & 23,31 \\
\hline Agrícola & 28,74 & 30,19 \\
\hline Otros $(2)$ & 12,03 & 12,00 \\
\hline \multicolumn{3}{|l|}{ Formalidad (3) } \\
\hline Formal (> 5 empleados) & 34,30 & 34,66 \\
\hline Números de observaciones & 24174 & 22834 \\
\hline
\end{tabular}

(2) Incluye trabajadores del hogar con y sin remuneración y empleados domésticos.

(3) Tamaño es usada como proxy. La variable tamaño está definida en función del número de empleados. Una empresa grande es aquella con 5 o más empleados y se asume como formal. 
Cuadro 2a - Estadística descriptiva de las variables Ingreso, Escolaridad y Experiencia.

\begin{tabular}{|l|c|c|c|c|}
\hline \multicolumn{5}{|c|}{ 1995 } \\
\hline Variables & Ingreso (4) & Escolaridad & Ingreso (5) & Escolaridad \\
Media & 166304,2 & 5,28 & 124230,99 & 5,58 \\
Por quintiles & & & & \\
Q1 & & & 9412,86 & 3,52 \\
& 15064,03 & 3,23 & $(8277,39)$ & $(3,45)$ \\
Q2 & $(11310,95)$ & $(3,22)$ & 41470,44 & 4,30 \\
& 51948,59 & 3,99 & $(9847,35)$ & $(3,87)$ \\
Q3 & $(10397,29)$ & $(3,66)$ & 73874,26 & 5,08 \\
Q4 & 96026,69 & 4,82 & $(10366,66)$ & $(4,08)$ \\
& $(14848,2)$ & $(4,17)$ & 128294,54 & 6,46 \\
Q5 & 163028,8 & 6,07 & $(22747,62)$ & $(4,67)$ \\
& $(27114,97)$ & $(4,69)$ & 390961,19 & 8,86 \\
& 497233,4 & 8,18 & $(408004,38)$ & $(5,54)$ \\
\hline
\end{tabular}
(4) Ingreso laboral individual.
(5) Cifras en sucres de 1995. 
Cuadro 3a - Resultados de las estimaciones de la ecuación de Ingresos (Especificaciones corregidas por el sesgo de selección).

\begin{tabular}{|c|c|c|c|c|}
\hline \multirow{3}{*}{$\begin{array}{l}\text { Ecuación de ingresos } \\
\text { Constante }\end{array}$} & \multicolumn{2}{|c|}{1995} & \multicolumn{2}{|c|}{1998} \\
\hline & & & & \\
\hline & $\begin{array}{c}12,064 \\
(138,50)\end{array}$ & $\begin{array}{c}11,879 \\
(122,77)\end{array}$ & $\begin{array}{l}12,625 \\
(118,21)\end{array}$ & $\begin{array}{c}12,328 \\
(108,10)\end{array}$ \\
\hline Escolaridad & $\begin{array}{c}0,081 \\
(21,94)\end{array}$ & $\begin{array}{c}0,082 \\
(21,73)\end{array}$ & $\begin{array}{c}0,079 \\
(20,20)\end{array}$ & $\begin{array}{c}0,080 \\
(20,22)\end{array}$ \\
\hline Experiencia & $\begin{array}{l}0,030 \\
(6,90)\end{array}$ & $\begin{array}{l}0,029 \\
(6,63)\end{array}$ & $\begin{array}{l}0,036 \\
(7,08)\end{array}$ & $\begin{array}{l}0,037 \\
(7,42)\end{array}$ \\
\hline Experiencia 2 & $\begin{array}{c}-0,00044 \\
(-5,72)\end{array}$ & $\begin{array}{c}-0,00043 \\
(-5,54)\end{array}$ & $\begin{array}{c}-0,00056 \\
(6,65)\end{array}$ & $\begin{array}{c}-0,00058 \\
(6,94)\end{array}$ \\
\hline Género & $\begin{array}{c}-0,566 \\
(-19,73)\end{array}$ & $\begin{array}{l}-0,505 \\
(-16,71)\end{array}$ & $\begin{array}{l}-0,536 \\
(16,95)\end{array}$ & $\begin{array}{l}-0,472 \\
(14,37)\end{array}$ \\
\hline Trabajador & $-0,644$ & $-0,468$ & $-0,614$ & $-0,376$ \\
\hline Agrícola & $(19,17)$ & $(-11,16)$ & $(18,93)$ & $(8,35)$ \\
\hline Sector Público & $\begin{array}{l}0,050 \\
(1,61)\end{array}$ & $\begin{array}{l}0,089 \\
(2,63)\end{array}$ & $\begin{array}{l}0,195 \\
(6,24)\end{array}$ & $\begin{array}{l}0,269 \\
(7,85)\end{array}$ \\
\hline Educación padre & $\begin{array}{l}0,392 \\
(5,24)\end{array}$ & $\begin{array}{l}0,372 \\
(5,02)\end{array}$ & $\begin{array}{l}0,404 \\
(6,22)\end{array}$ & $\begin{array}{l}0,379 \\
(5,96)\end{array}$ \\
\hline Educación madre & $\begin{array}{l}0,033 \\
(0,29)\end{array}$ & $\begin{array}{l}0,037 \\
(0,33)\end{array}$ & $\begin{array}{c}0,337 \\
(0,337)\end{array}$ & $\begin{array}{c}0,353 \\
(0,353)\end{array}$ \\
\hline Tamaño fïma & $\begin{array}{c}-0,373 \\
(-16,37)\end{array}$ & $\begin{array}{c}-0,381 \\
(-16,48)\end{array}$ & $\begin{array}{c}-0394 \\
(16,50)\end{array}$ & $\begin{array}{l}-0,405 \\
(16,43)\end{array}$ \\
\hline Etnia & $\begin{array}{l}-0,282 \\
(-3,68)\end{array}$ & $\begin{array}{l}-0,275 \\
(-3,60)\end{array}$ & $\begin{array}{c}-0,289 \\
(4,10)\end{array}$ & $\begin{array}{c}-0,274 \\
(3,89)\end{array}$ \\
\hline Ramas de actividad & & & & \\
\hline Manufactura & & $\begin{array}{c}0,044 \\
(0,60)\end{array}$ & & $\begin{array}{l}0,184 \\
(2,39)\end{array}$ \\
\hline Minería & & $\begin{array}{l}0,048 \\
(0,96)\end{array}$ & & $\begin{array}{l}0,241 \\
(4,16)\end{array}$ \\
\hline Electricidad y otros & & 0,303 & & 0,321 \\
\hline Servicios & & $(7,67)$ & & $(6,72)$ \\
\hline Construcción & & $\begin{array}{l}0,252 \\
(6,83)\end{array}$ & & $\begin{array}{l}0,263 \\
(6,53)\end{array}$ \\
\hline Comercio & & $\begin{array}{l}0,436 \\
(9,15)\end{array}$ & & $\begin{array}{c}0,568 \\
(11,62)\end{array}$ \\
\hline Transporte & & $\begin{array}{l}0,191 \\
(3,81)\end{array}$ & & $\begin{array}{l}0,249 \\
(5,55)\end{array}$ \\
\hline Servicios varios & & $\begin{array}{c}0,008 \\
(0,20)\end{array}$ & & $\begin{array}{l}0,168 \\
(3,57)\end{array}$ \\
\hline
\end{tabular}




\begin{tabular}{|l|c|c|c|c|}
\hline \multirow{4}{*}{ Ecuación de selección } & \multicolumn{2}{|c|}{1995} & \multicolumn{2}{c|}{1998} \\
\cline { 2 - 5 } Escolaridad & & & & \\
& 0,028 & 0,028 & 0,029 & 0,029 \\
Estado civil & $(8,58)$ & $(8,55)$ & $(8,37)$ & $(8,37)$ \\
Edad & $-0,155$ & $-0,156$ & $-0,199$ & $-0,201$ \\
& $(-4,55)$ & $(-4,59)$ & $(5,48)$ & $(5,49)$ \\
Edad 2 & 0,156 & 0,156 & 0,159 & 0,159 \\
Jefe de familia & $(30,11)$ & $(30,09)$ & $(27,80)$ & $(27,79)$ \\
Hijo chico & $-0,002$ & $-0,002$ & $-0,002$ & $-0,002$ \\
Ingreso laboral & $(-28,20)$ & $(-28,18)$ & $(25,14)$ & $(25,19)$ \\
& 1,296 & 1,296 & 1,208 & 1,211 \\
Constante & $(34,47)$ & $(34,48)$ & $(24,55)$ & $(24,89)$ \\
& 0,273 & 0,273 & 0,347 & 0,347 \\
Observaciones & $(6,05)$ & $(6,05)$ & $(7,70)$ & $(7,67)$ \\
& $-8,36 \mathrm{e}-07$ & $-8,37 \mathrm{e}-07$ & $-1,70 \mathrm{e}-07$ & $-1,76 \mathrm{e}-07$ \\
& $(-4,62)$ & $(-4,61)$ & $(2,45)$ & $(2,51)$ \\
& $-3,155$ & $-3,154$ & $-3,274$ & $-3,274$ \\
& $(-34,37)$ & $(-34,33)$ & $(33,39)$ & $(33,35)$ \\
& 16837 & 16837 & 15951 & 15951 \\
\hline
\end{tabular}

Cuadro 4a - Contribuciones a la desigualdad - Metodología de Fields 1995 y 1998 (6).

\begin{tabular}{|l|c|c|c|c|}
\hline \multirow{2}{*}{\multicolumn{1}{c|}{ Variables }} & \multicolumn{2}{|c}{1995} & \multicolumn{2}{c|}{1998} \\
\cline { 2 - 5 } & $\mathbf{( 1 )}$ & $\mathbf{( 2 )}$ & $\mathbf{( 1 )}$ & $\mathbf{( 2 )}$ \\
\cline { 2 - 5 } \% Varianza del Ingreso no explicada & 64,99 & 64,81 & 60,22 & 60,98 \\
\% Varianza del Ingreso explicada & 35,01 & 35,19 & 39,78 & 39,02 \\
& & & & \\
Educación & 14,60 & 15,38 & 16,00 & 16,05 \\
Experiencia & 1,00 & 0,78 & 2,76 & 2,84 \\
Género & 5,18 & 4,53 & 4,42 & 3,90 \\
Etnia & 0,48 & 0,46 & 0,64 & 0,61 \\
Educación padres & 0,73 & 0,70 & 1,23 & 1,18 \\
Sector público & 0,15 & 0,44 & 0,79 & 1,00 \\
Sector agrícola & 7,97 & 6,81 & 8,22 & 4,70 \\
Tamaño & 4,90 & 5,01 & 5,72 & 5,87 \\
Total ramas de actividad & \multicolumn{4}{|c}{} \\
\end{tabular}

(6) El computo de las contribuciones se realizó a base del modelo de ingresos corregido por sesgo de selección (Heckman). 
Cuadro 5a - Contribuciones a los cambios en la desigualdad de los factores experiencia y pertenencia al sector agrícola entre 1995 y 1998.

\begin{tabular}{|l|c|c|c|}
\hline & Experiencia & Experiencia 2 & $\begin{array}{c}\text { Pertenencia al } \\
\text { sector agrícola }\end{array}$ \\
\cline { 2 - 4 } Beta factor & $90 \%$ & $83 \%$ & $81 \%$ \\
Correlación (factor, In ingreso) & $10 \%$ & $13 \%$ & $81 \%$ \\
Stdv (Factor) & $11 \%$ & $11 \%$ & $-13 \%$ \\
Stdv (In ingreso) & $-11 \%$ & $-7 \%$ & $-50 \%$ \\
\hline
\end{tabular}

Cuadro 6a - Contribuciones a la desigualdad - Metodología de Shorrocks 1995 y 1998.

\begin{tabular}{|c|c|c|c|c|c|c|}
\hline \multicolumn{4}{|c|}{1995} & \multicolumn{3}{|c|}{1998} \\
\hline Fuentes & $\begin{array}{c}100 *{ }_{\text {Skv }} \\
\text { (1) }\end{array}$ & $\begin{array}{c}100 *{ }_{\text {Skg }} \\
\text { (2) }\end{array}$ & $\begin{array}{c}100 *{ }_{\text {Skf }} \\
\text { (3) }\end{array}$ & $\begin{array}{c}100 *{ }_{\text {Skv }} \\
(4)\end{array}$ & $\begin{array}{c}100 *{ }_{\text {Skg }} \\
\text { (5) }\end{array}$ & $\begin{array}{l}100 *{ }_{\text {Skf }} \\
(6)\end{array}$ \\
\hline \multicolumn{7}{|c|}{ Ingreso laboral } \\
\hline Jefe de familia & 51,61 & 47,96 & 53,40 & 50,96 & 53,29 & 56,62 \\
\hline Cónyuge & 15,75 & 15,23 & 12,94 & 18,49 & 13,74 & 11,29 \\
\hline Otros miembros & 19,04 & 23,01 & 21,17 & 16,28 & 21,53 & 20,96 \\
\hline \multicolumn{7}{|c|}{ Ingreso no laboral } \\
\hline Ayuda familiar & 1,93 & 2,75 & 3,86 & 4,24 & 4,33 & 5,20 \\
\hline Donaciones & $-0,001$ & 0,09 & 0,15 & 0,23 & 0,22 & 0,14 \\
\hline Impuestos & $-0,76$ & $-0,65$ & $-0,57$ & $-0,61$ & $-0,54$ & $-0,82$ \\
\hline Pensiones & $0,-83$ & 2,06 & 2,21 & 1,52 & 2,44 & 2,43 \\
\hline Rentas del Capital & 6,85 & 5,90 & 4,56 & 8,25 & 5,06 & 3,60 \\
\hline Otros ingresos & 4,76 & 3,65 & 2,29 & 0,63 & 0,82 & 0,57 \\
\hline Total & 100,00 & 100,00 & 100,00 & 100,00 & 100,00 & 100,00 \\
\hline $\mathrm{N}^{\circ}$ observaciones & & 5084 & & & 5019 & \\
\hline
\end{tabular}

skv: proporción de desigualdad explicada por la descomposición de la varianza

skg: proporción de desigualdad explicada por la descomposición del gini

skf: proporciones en el ingreso del factor correspondiente 
Cuadro 7a - Tasas de participación fem. por familia agrícola y quintiles de ingresos.

\begin{tabular}{|l|c|c|c|c|c|c|}
\hline & & $\mathbf{1 9 9 5}$ & & & $\mathbf{1 9 9 8}$ & \\
\hline & Agrícola & No agrícola & Total & Agrícola & No agrícola & Total \\
\hline 1er. & 33,69 & 29,39 & 40,91 & 36,81 & 26,02 & 33,18 \\
2do. & 25,71 & 30,67 & 40,76 & 32,68 & 29,75 & 31,03 \\
3er. & 33,23 & 31,29 & 43,84 & 38,36 & 37,01 & 37,36 \\
4to. & 37,52 & 38,24 & 48,78 & 39,34 & 39,62 & 39,57 \\
5to. & 39,03 & 51,26 & 56,93 & 39,79 & 51,20 & 49,30 \\
\hline
\end{tabular}

Cuadro 8a - Contribuciones a la Desigualdad de Grupo No Agrícola.

\begin{tabular}{|l|c|c|c|c|c|c|}
\hline \multicolumn{7}{|c|}{ Contribución a la desigualdad Participación en el ingreso } \\
\hline Fuentes & $\mathbf{1 9 9 5}$ & $\mathbf{1 9 9 8}$ & Variación & $\mathbf{1 9 9 5}$ & $\mathbf{1 9 9 8}$ & Variación \\
\hline \multicolumn{7}{|c|}{ Ingreso Laboral } \\
\hline Jefe de familia & 57,98 & 58,87 & 0,89 & 60,97 & 63,60 & 2,63 \\
Cónyuge & 14,30 & 20,71 & 6,41 & 13,67 & 12,30 & $-1,37$ \\
Otros miembros & 14,38 & 12,08 & $-2,3$ & 14,46 & 15,24 & 0,78 \\
\hline \multicolumn{7}{|c|}{ Ingreso no laboral } \\
\hline Ayuda familiar & 1,76 & 3,83 & 2,07 & 2,61 & 4,04 & 1,43 \\
Donaciones & 0,001 & 0,26 & 0,259 & 0,05 & 0,15 & 0,1 \\
Impuestos & $-0,85$ & $-0,60$ & 0,25 & $-0,58$ & $-0,83$ & $-0,25$ \\
Pensiones & 0,33 & 0,95 & 0,62 & 1,26 & 1,35 & 0,09 \\
Rentas del Capital & 6,52 & 9,25 & 2,73 & 3,47 & 3,60 & 0,13 \\
Otros ingresos & 5,58 & 0,66 & $-4,92$ & 2,09 & 0,55 & $-1,54$ \\
Total & 100,00 & 100,00 & 100,00 & 100,00 & 100,00 & 100,00 \\
\hline
\end{tabular}

Cuadro 9a - Tasas de participación por género del individuo.

\begin{tabular}{|l|c|c|}
\hline & $\mathbf{1 9 9 5}$ & $\mathbf{1 9 9 8}$ \\
\cline { 2 - 3 } Mujeres & 49,10 & 51,06 \\
Hombres & 78,84 & 79,18 \\
Total & 63,67 & 64,91 \\
\hline
\end{tabular}


Cuadro 10a - Contribuciones a la Desigualdad de Grupo Jefe de Familia Hombre.

\begin{tabular}{|l|c|c|c|c|c|c|}
\hline \multicolumn{7}{|c|}{ Contribución a la desigualdad Participación en el ingreso } \\
\hline Fuentes & $\mathbf{1 9 9 5}$ & $\mathbf{1 9 9 8}$ & Variación & $\mathbf{1 9 9 5}$ & $\mathbf{1 9 9 8}$ & Variación \\
\hline \multicolumn{7}{|c|}{ Ingreso Laboral } \\
\hline Jefe de familia & 54,09 & 54,20 & 0,11 & 57,07 & 61,04 & 3,97 \\
Cónyuge & 17,09 & 19,91 & 2,82 & 14,53 & 12,51 & $-2,02$ \\
Otros miembros & 16,89 & 14,29 & $-2,6$ & 18,40 & 18,78 & 0,38 \\
\hline \multicolumn{7}{|c|}{ Ingreso no laboral } \\
\hline Ayuda familiar & 1,10 & 0,93 & $-0,17$ & 2,28 & 2,72 & 0,44 \\
Donaciones & $-0,001$ & 0,25 & 0,251 & 0,11 & 0,16 & 0,05 \\
Impuestos & $-0,83$ & $-0,61$ & 0,22 & $-0,62$ & $-0,79$ & $-0,17$ \\
Pensiones & 0,78 & 1,19 & 0,41 & 1,86 & 2,07 & 0,21 \\
Rentas del Capital & 6,85 & 9,15 & 2,3 & 4,11 & 3,54 & $-0,57$ \\
Otros ingresos & 4,03 & 0,68 & $-3,35$ & 2,25 & 0,57 & $-1,68$ \\
& \multicolumn{7}{|c|}{} & & & \\
\hline
\end{tabular}

Cuadro 11a - Contribuciones a la Desigualdad de Grupo Jefe Capacitado.

\begin{tabular}{|l|c|c|c|c|c|c|}
\hline & Contribución a la desigualdad & Participación en el ingreso \\
\hline Fuentes & $\mathbf{1 9 9 5}$ & $\mathbf{1 9 9 8}$ & Variación & $\mathbf{1 9 9 5}$ & $\mathbf{1 9 9 8}$ & Variación \\
\hline \multicolumn{7}{|c|}{ Ingreso Laboral } \\
\hline Jefe de familia & 52,73 & 50,78 & $-1,95$ & 56,15 & 59,48 & 3,33 \\
Cónyuge & 16,21 & 19,57 & 3,36 & 16,0 & 12,60 & $-3,49$ \\
Otros miembros & 17,98 & 14,91 & $-3,07$ & 17,02 & 16,36 & $-0,66$ \\
\hline \multicolumn{7}{|c|}{ Ingreso no laboral } \\
\hline Ayuda familiar & 1,82 & 4,20 & 2,38 & 3,57 & 4,98 & 1,41 \\
Donaciones & $-0,004$ & 0,24 & 0,244 & 0,13 & 0,16 & 0,03 \\
Impuestos & $-0,84$ & $-0,61$ & 0,23 & $-0,67$ & $-0,92$ & $-0,25$ \\
Pensiones & 0,72 & 1,44 & 0,72 & 2,40 & 2,66 & 0,26 \\
Rentas del Capital & 6,01 & 8,82 & 2,81 & 4,68 & 4,06 & $-0,62$ \\
Otros ingresos & 5,37 & 0,64 & $-4,73$ & 2,63 & 0,63 & -2 \\
Total & 100,00 & 100,00 & 0,00 & 100,00 & 100,00 & 0,00 \\
\hline
\end{tabular}


Cuadro 12a - Contribuciones a la Desigualdad de Grupo Empleo Formal.

\begin{tabular}{|l|c|c|c|c|c|c|}
\hline & Contribución a la desigualdad & \multicolumn{2}{c|}{ Participación en el ingreso } \\
\hline Fuentes & $\mathbf{1 9 9 5}$ & $\mathbf{1 9 9 8}$ & Variación & $\mathbf{1 9 9 5}$ & $\mathbf{1 9 9 8}$ & Variación \\
\hline \multicolumn{7}{|c|}{ Ingreso Laboral } \\
\hline Jefe de familia & 49,08 & 50,76 & 1,68 & 49,98 & 54,47 & 4,49 \\
Cónyuge & 16,20 & 20,31 & 4,11 & 13,33 & 12,37 & $-0,96$ \\
Otros miembros & 19,12 & 16,96 & $-2,16$ & 21,47 & 21,34 & $-0,13$ \\
\hline \multicolumn{7}{|c|}{ Ingreso no laboral } \\
\hline Ayuda familiar & 1,75 & 2,13 & 0,38 & 4,21 & 5,09 & 0,88 \\
Donaciones & $-0,03$ & 0,27 & 0,3 & 0,22 & 0,20 & $-0,02$ \\
Impuestos & $-0,92$ & $-0,78$ & 0,14 & $-0,67$ & $-0,97$ & $-0,3$ \\
Pensiones & 0,94 & 1,57 & 0,63 & 2,88 & 3,12 & 0,24 \\
Rentas del Capital & 7,18 & 8,18 & 1 & 5,45 & 3,87 & $-1,58$ \\
Otros ingresos & 6,68 & 0,59 & $-6,09$ & 3,15 & 0,51 & $-2,64$ \\
Total & 100,00 & 100,00 & 0,00 & 100,00 & 100,00 & 0,00 \\
\hline
\end{tabular}

Cuadro 13a - Tasas de desempleo y subempleo en Ecuador en el período 1995-2000.

\begin{tabular}{|c|c|c|}
\hline Año & Tasa de desempleo & Tasa de subempleo \\
\hline 1995 & $8 \%$ & $45 \%$ \\
1996 & $9 \%$ & $43 \%$ \\
1997 & $9 \%$ & $40 \%$ \\
1998 & $12 \%$ & $46 \%$ \\
1999 & $14 \%$ & $57 \%$ \\
2000 & $9 \%$ & $66 \%$ \\
\hline
\end{tabular}

Fuente: Encuestas Urbanas de Empleo, 1991-1997. 